\title{
Regio- and Stereoselective Aminopentadienylation of Carbonyl Compounds
}

Irene Bosque, ${ }^{a}$ Emine Bagdatli, ${ }^{\mathrm{b}}$ Francisco Foubelo, ${ }^{\mathrm{a}}$ and Jose C. Gonzalez-Gomez*,a

${ }^{a}$ Departamento de Química Orgánica, Facultad de Ciencias and Instituto de Síntesis

Orgánica (ISO), Universidad de Alicante, Apdo. 99, 03080 Alicante, Spain.

${ }^{\mathrm{b}}$ Current address: Faculty of Science and Arts, Cumhuriyet Campus, Ordu University, 52200 Ordu, Turkey.

*E-mail: josecarlos.gonzalez@ua.es

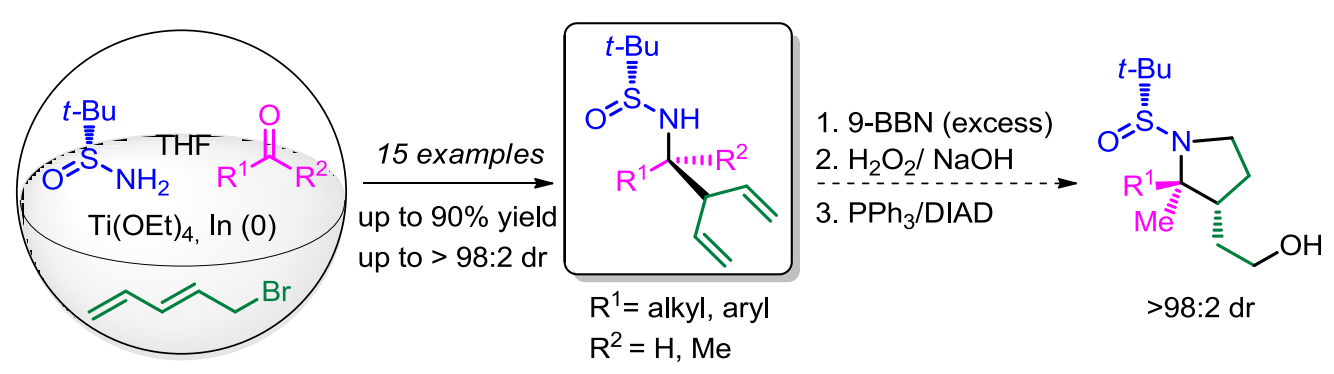

ABSTRACT: A simple and robust protocol is detailed for the preparation of enantioenriched $\alpha$-substituted(1,4-pentadien-3-yl)amine derivatives. The methodology involves the addition of an in-situ formed pentadienyl indium reagent to chiral tertbutylsulfinimines, previously formed in the same pot. The addition takes place with excellent $\gamma$-regio and diastereoselectivity for a wide range of carbonyl compounds, including $\alpha$-unsubstituted aldehydes and methyl alkyl ketones. The catalytic hydrogenation of the sulfinamines obtained provides a convenient access to chiral $\alpha$ substituted (3-pentyl)amines. The hydroboration-oxidation of the $\alpha$-(1,4-pentadien-3yl)amine derivatives, followed by a cyclization under Mitsunobu conditions, takes place with an excellent diastereoselectivity governed by the chiral sulfinyl group. 


\section{INTRODUCTION}

Pentadienylmetals can suffer from metallotropic 1,3-or 1,5-rearrangements and upon reaction with electrophiles can give rise to three possible regioisomers: the $\alpha-, \gamma$ - and $\varepsilon$ adducts. The addition to aldehydes or ketones of pentadienyl reagents of $\mathrm{Mg},{ }^{1} \mathrm{Be},{ }^{2} \mathrm{Zn},{ }^{3}$ $\mathrm{Sn},{ }^{4}, \mathrm{Si}^{5}{ }^{5}$ and $\mathrm{B}^{6}$ has been examined under different conditions. The regioselectivity differs from one case to another but the $\gamma$-adduct is the main product in most cases. Many of the protocols examined are limited by the use of hazardous or moisture sensitive reagents, which complicates their manipulation or makes these procedures poorly reliable. Importantly, the alcohols obtained in the $\gamma$-pentadienylation of carbonyl compounds have proven to be valuable building blocks in the synthesis of more complex molecules. ${ }^{7}$

The pentadienylation of imines, using tributylpentadienyltin and Lewis acids (i. e. $\left.\mathrm{InCl}_{3}\right)$ as additives was studied by the group of Nishigaichi. ${ }^{8}$ In their work, the authors found that $N$-phenyl imines afford the $\varepsilon$-adduct as the only regioisomer, presumably by Lewis acid activation of the imine and nucleophilic attack of the pentadienyltin species through an acyclic transition state. Remarkably, with less basic $N$-tosyl imines, the only regioisomer isolated was the corresponding $\gamma$-adduct. The formation of this compound was explained by considering that after transmetallation, the resulting pentadienylindium intermediate coordinates to the iminic nitrogen and reacts through a cyclic transition state (Scheme 1).

Scheme 1. Addition of Tributylpentadienyltin Reagent to Imines Catalyzed by $\mathrm{InCl}_{3}$. 


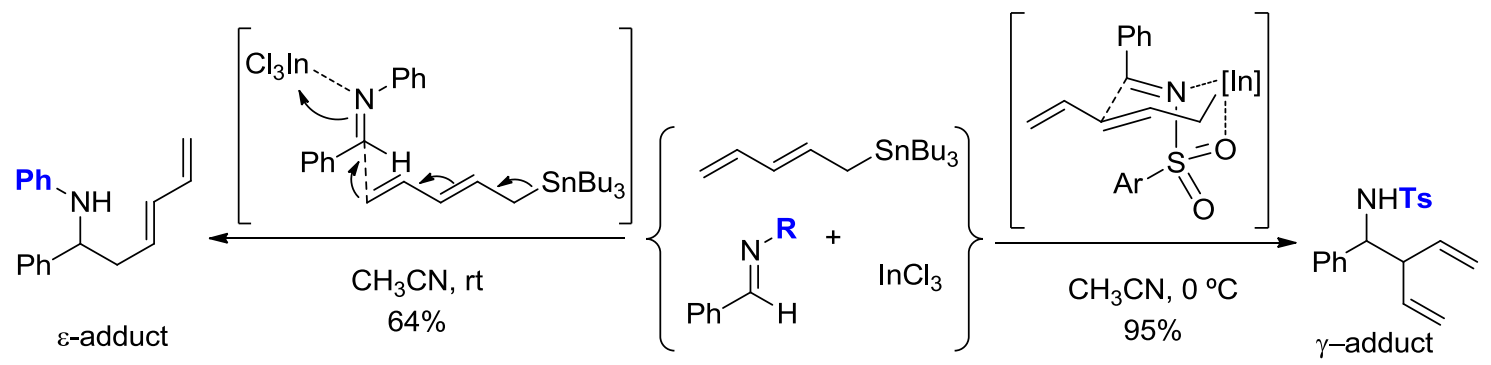

The indium-mediated Barbier-type reaction is a superior protocol to the above mentioned procedures due to its experimental simplicity and because toxic reagents are avoided. ${ }^{9}$ In this context Araki and co-workers examined the addition of 2,4-pentadienyl indium derivatives, under Barbier conditions, observing the exclusive formation of $\gamma$ adducts in the addition to carbonyl compounds. ${ }^{10}$ Soon after, the group of Fallis observed that in-situ formed 2,4-pentadienyl indium reacts smoothly with a range of carbonyl compounds, including $\alpha, \beta$-unsaturated aldehydes and ketones, in DMF or aqueous media also with excellent $\gamma$-selectivity. ${ }^{11}$

The development of new practical methodologies for the $\gamma$-regioselective addition of pentadienyl metal reagents to imines is driven by the potential of the corresponding adducts in the construction of more complex molecules. An elegant example was recently illustrated by the group of Martin during the synthesis of racemic Pseudotabersonine. ${ }^{12}$ This natural product was prepared from the adduct obtained by addition of a pentadienyl aluminium reagent to the corresponding aromatic imine (Scheme 2). Importantly, the same scaffold is present in other Aspidosperma alkaloids such as Aspidospermidine and Pandoline.

Scheme 2. Pentadienylation of Imines in the Construction of Natural Product Scaffolds. 


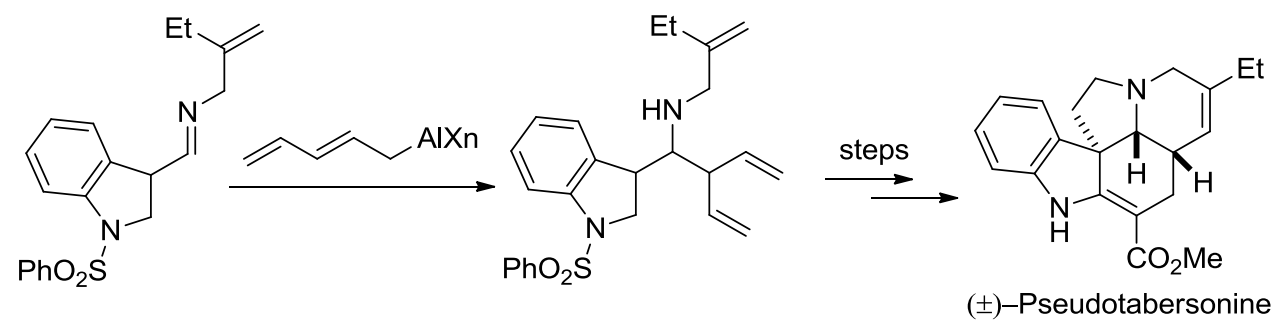

At the outset of this work, we were not aware of any stereocontrolled addition of pentadienyl indium reagent to imine derivatives. ${ }^{13}$ In this context we decided to expand the scope of our indium-mediated aminoallylation of aldehydes ${ }^{14}$ with chiral tertbutylsulfinamide ${ }^{15}$ by using a pentadienyl indium reagent, generated in situ. This approach would allow the regio- and stereoselective formation of chiral $\alpha$-substituted (1,4-pentadien-3-yl)amines, which could act as building blocks for other interesting target molecules.

\section{RESULTS AND DISCUSSION}

The one-pot protocol developed in our research group for the $\alpha$-aminoallylation of aldehydes was implemented to prepare several enantioenriched $\alpha$-substituted-(1,4pentadien-3-yl)amines. In this case, the required pentadienyl bromide was prepared by reaction of commercially available penta-1,4-dien-3-ol with $\mathrm{PBr}_{3}$ in diethyl ether at $0{ }^{\circ} \mathrm{C}$ for $1 \mathrm{~h}$. Our methodology involves the formation of the corresponding imine by condensation of an aldehyde with enantiopure $\mathrm{N}$-tert-butylsulfinamide in the presence of $\mathrm{Ti}(\mathrm{OEt})_{4}$ and indium powder at room temperature. After $1 \mathrm{~h}$, the prepared pentadienyl bromide was added to the reaction mixture and the temperature was increased to $60{ }^{\circ} \mathrm{C}$. Under these conditions, a range of aldehydes was examined (Table $1)$. 
Benzaldehyde afforded the corresponding $\gamma$-adduct $4 \mathbf{a}$ in only $14 \%$ yield as an inseparable mixture of 9:1 diastereoisomers. ${ }^{16}$ Better yields and good diastereoselectivities were achieved with more electron-deficient aromatic substrates like 3-, or 4-chlorobenzaldehyde $(\mathbf{2 b}, \mathbf{2 c})$, although the bulkier 2-chlorobenzaldehyde afforded product $\mathbf{4 d}$ in poorer yield. The $\alpha$-branched cyclohexanecarbaldehyde gave a 74:26 mixture of $\gamma / \alpha$ adducts. The major $\gamma$-regioisomer $\mathbf{4 e}$ was isolated in good yield after column chromatography. Importantly, the configuration at the newly formed stereogenic center in $\mathbf{4 e}$ was confirmed to be $(R)$ by X-ray crystal diffraction analysis (see supporting information), which fits with our working model that predicts addition of the allylic reagent onto the $s i$-face of the $\left(R_{\mathrm{S}}\right)$-sulfinimine (Table 1). We were pleased to observe that $\alpha$-unsubstituted aldehydes $(\mathbf{2 f}-\mathbf{2 l})$, which are more challenging substrates with other allylic organometallic species due to their easy enolization, reacted well with this protocol. Notably, the presence of halogen atoms was tolerated in the substrates (2b-2d, 2g) and only $\gamma$-adducts were isolated in good yields and with excellent diasteroselectivity. The 1,2-addition product $(\mathbf{4 j})$ was isolated exclusively when cinnamaldehyde was examined. Furthermore when $(S)$-citronellal was examined with either enantiomer of the $\mathrm{N}$-tert-butylsulfinamide, products $\mathbf{4 k}$ and $\mathbf{4 l}$ were both obtained in very good yields and diastereoselectivities. The configuration at the newly stereogenic center formed was controlled by the chiral sulfur atom without remarkable matched or mismatched effect.

Table 1. Aminopentadienylation of Aldehydes 


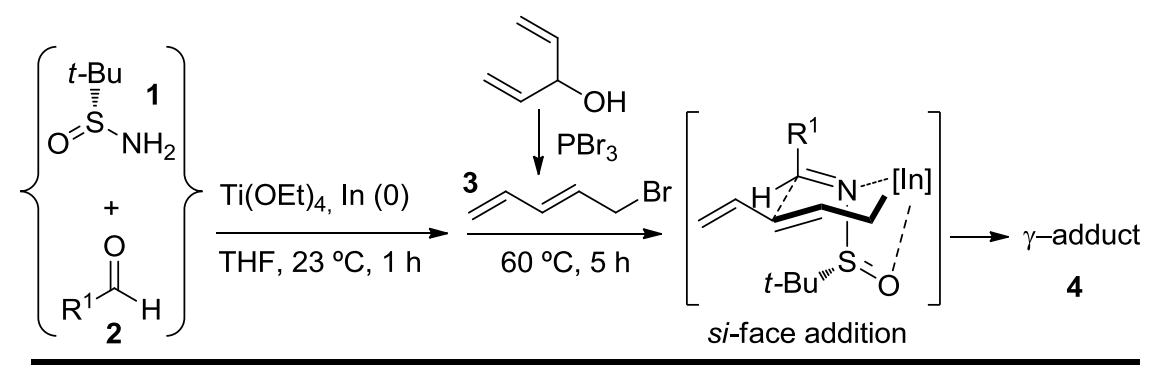

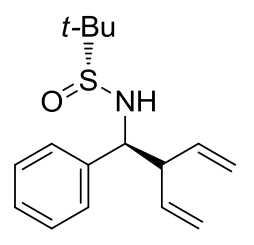

4a: $14 \%, 90: 10 d r$<smiles>C=CC(C=C)[C@H](NS([13CH])=O)C1CCCCC1</smiles>

4e: $55 \%{ }^{a}>98: 2 d r$<smiles>C=CC(C=C)[C@H](NS([10CH2])=O)c1ccc(Cl)cc1</smiles>

4b: $66 \%$, >98:2 dr

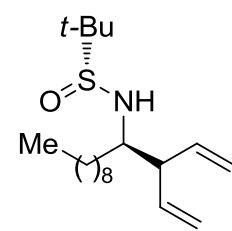

4f: $90 \%, 98: 2 d r$<smiles>C=CC(C=C)[C@H](CCc1ccccc1)NS([13CH])=O</smiles>

$4 i:^{b} 85 \%, 97: 3 d r$<smiles>C=CC(C=C)C(/C=C/c1ccccc1)NS(=O)C(C)(C)C</smiles>

4j: $72 \%, 97: 3 d r$<smiles>C=CC(C=C)C(NS(=O)[14C](C)(C)C)c1cccc(Cl)c1</smiles>

4c: $83 \%, 90: 10 \mathrm{dr}$<smiles>C=CC(C=C)C(CCCCBr)NS(=O)C(C)(C)C</smiles>

4g: $73 \%,>98: 2 \mathrm{dr}$<smiles>C=CC(C=C)C(CC(C)CCC=C(C)C)NS(=O)C(C)(C)C</smiles>

4k: $86 \%,>97: 3{d r^{c}}^{c}$<smiles>C=CC(C=C)C(NS(=O)[18OH])c1ccccc1Cl</smiles>

4d: $21 \%,>98: 2 \mathrm{dr}$<smiles>C=CC(C=C)C(Cc1ccccc1)NS(=O)C(C)(C)C</smiles>

4h: ${ }^{\text {b }} 70 \%, 98: 2 \mathrm{dr}$<smiles>C=CC(CC(C)CC/C=C(/[N+](=O)[O-])C(C)(C)C)[C@H](C=C)NS(=O)C(C)(C)C</smiles>

$4 l^{\mathrm{d}} 84 \%,>97: 3 \mathrm{dr}^{\mathrm{c}}$

Isolated yields and diastereomeric ratios ( ${ }^{1} \mathrm{H}-\mathrm{NMR}$ spectroscopy) after column chromatography are shown. ${ }^{\text {a }}$ The crude reaction mixture showed a 74:26 mixture of $\gamma$-and $\alpha$-adducts. ${ }^{\mathrm{b}}$ ent- $\mathbf{- 4 h}$ and ent-4i were also synthesized using ent-1 ${ }^{\mathrm{c}}$ Signals corresponding to diastereoisomers are not observed in the ${ }^{13} \mathrm{C}$ NMR spectra. ${ }^{\mathrm{d}} \mathrm{In}$ this case, ent-1 was used.

Encouraged by the good results reported for the indium-mediated allylation of tertbutylsulfinyl ketimines, ${ }^{17}$ we decided to apply our pentadienylation methodology to ketones (Scheme 3). In this case the formation of the corresponding ketimines required 
an increase of the temperature to $60{ }^{\circ} \mathrm{C}$ and reaction time to $8 \mathrm{~h}$, whereupon 5-bromo1,3-pentadiene was added. That the indium powder was still active after the imine formation, confirms the stability of the metal in the presence of moisture and/or ethanol at $60{ }^{\circ} \mathrm{C}$. Aliphatic methyl ketones examined under these conditions (5a-5c), afforded $\gamma$ adducts exclusively (6a-6c) in good yields and with excellent diastereoselectivities. ${ }^{18}$

\section{Scheme 3. Aminopentadienylation of Methyl Alkyl Ketones}
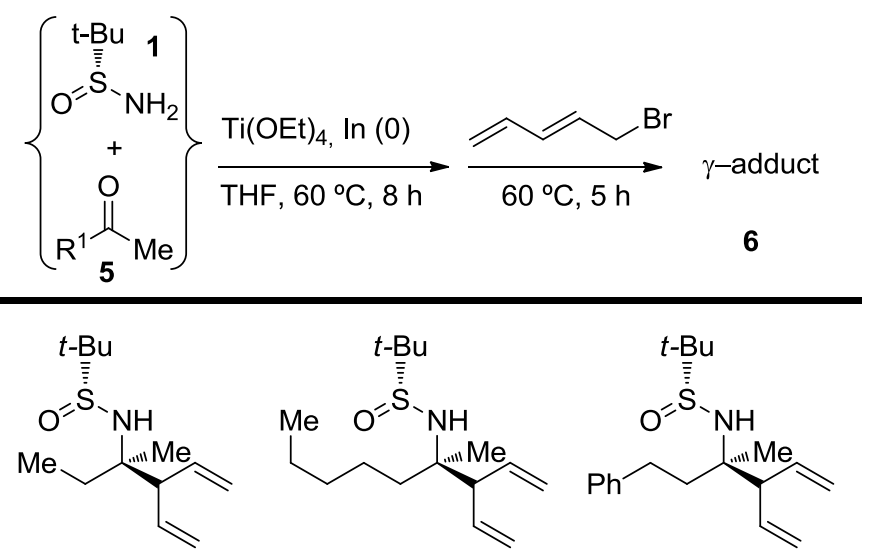

$\begin{array}{lll}\text { 6a: } 62 \%, 97: 3 \mathrm{dr} & \text { 6b: } 65 \%,>98: 2 \mathrm{dr} & \text { 6c: } 72 \%,>98: 2 \mathrm{dr}\end{array}$

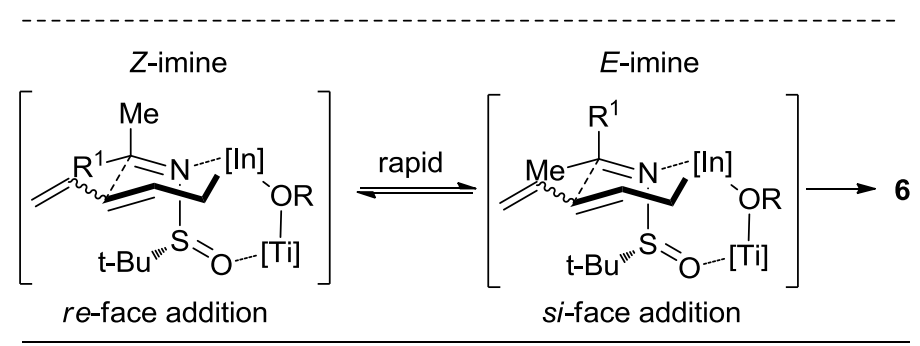

Isolated yields and diastereomeric ratios (determined by ${ }^{1} \mathrm{H}-\mathrm{NMR} \quad$ spectroscopy) after column chromatography are shown.

In order to evaluate the efficiency of this one-pot protocol we isolated the tertbutylsulfinyl imine of 2-heptanone (72\%) and submitted this to indium-mediated pentadienylation in THF. Under these conditions compound $\mathbf{6 b}$ was isolated in only $44 \%$ yield (32\% over two steps), being recovered 2-heptanone as the major side-product from a competitive hydrolytic process. We thus reasoned that the presence of $\operatorname{Ti}(\mathrm{OEt})_{4}$ 
improved the conversion of the intermediate imine by minimizing its hydrolysis. Moreover, the presence of $\mathrm{Ti}(\mathrm{IV})$ could also accelerate the pentadienylation process versus hydrolysis. In Scheme 3 we proposed a hypothetical more stable [4.4.0]-bicyclic transition state where the indium metal is coordinated to an alkoxy ligand acting as a bridge with a titanium center bonded to the oxygen atom of the sulfinyl group. The combination of $\mathrm{In}(\mathrm{III})$ and $\mathrm{Ti}(\mathrm{IV})$ in the same transition state might account for a more efficient Lewis acid activation. Importantly, we have found the same degree and sense of diastereoselection for the one-pot methodology and the two steps procedure. More importantly, while the intermediate imine was isolated as an 83:17 mixture of $E / Z$ isomers, compound $\mathbf{6 b}$ was obtained as a single isomer. Consequently, we reasoned that a dynamic kinetic resolution takes place where the $E / Z$ imines can rapidly interconvert in the presence of Lewis acids. ${ }^{19}$ The major diastereoisomer is formed from the addition of the pentadienyl indium reagent onto the si-face of the $\left(R_{\mathrm{S}}, E\right)$-imine, as previously observed in the two-steps protocol..$^{20,21}$

At this point we decided to explore some synthetic applications of the obtained enantioenriched pentadienyl amines. Hydrogenation of both double bonds was accomplished for substrates $4 \mathbf{f}$ and $\mathbf{6 a}$ using $\mathrm{PtO}_{2}$ as a catalyst. The sulfinyl group remained intact under these reaction conditions thereby avoiding the deprotection of the amine functionality. ${ }^{22}$ The corresponding amines $\mathbf{7}$ and $\mathbf{8}$ were obtained in excellent yields without any detectable epimerization (Scheme 4). To the best of our knowledge, chiral amines $\alpha$-substituted with a 3-pentyl moiety have not been reported so far. It is worth noting that a direct addition of 3-pentyl organometallic reagents to imines would be sterically disfavored and reduction or other processes related to single electron transfers are more reasonable in these cases (i.e. pinacol like coupling reactions).

\section{Scheme 4. Catalytic Hydrogenation of Pentadienyl Amines $4 f$ and 6a}




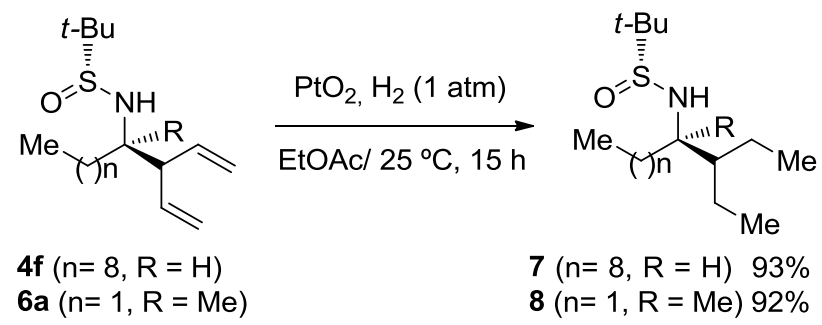

Given the occurrence of pyrrolidines in natural and synthetic bioactive compounds we consider of interest to develop a new entry to stereodefined 2,2,3-trisubstituted pyrrolidines. With this in mind we submitted $\alpha$-substituted pentadienyl amines $\mathbf{6 b}$ and 6c to a hydroboration/oxidation sequence using an excess of 9borabicyclo[3.3.1]nonane (9-BBN). The corresponding diols (9b and 9c, Scheme 5) were obtained in good yields and submitted to Mitsunobu reaction conditions to explore the differentiation of the diastereotopic hydroxyethyl groups upon cyclization. ${ }^{23} \mathrm{We}$ were pleased to observe that the corresponding pyrrolidines (10b and $\mathbf{1 0 c}$ ) were obtained with excellent diastereoselectivities, and isolated in very good yields as single isomers after column chromatography. ${ }^{24}$ To elucidate the configuration of the new stereocenter, compound 10c was transformed by conventional methods into the more rigid benzoyl derivative 11. After the assignment of all signals of the ${ }^{1} \mathrm{H}-\mathrm{NMR}$ spectra of compound 11 (COSY and HSQC experiments were used), relevant NOEs were identified that clearly indicated a trans-relationship between the methine proton and the methyl group. For a better understanding of this diastereoselective cyclization we removed the chirality of the sulfinyl group by oxidation with $m$-CPBA and submitted the obtained sulfonamide 12 to the same Mitsunobu reaction conditions. This reaction afforded a 1:1 diastereomeric mixture of pyrrolidines $\mathbf{1 3} / \mathbf{1 4}$, accompanied by a tetrahydropyran byproduct $\mathbf{1 5}$ (see experimental section). Oxidation of pyrrolidine $\mathbf{1 0 b}$ took place smoothly to afford pyrrolidine $\mathbf{1 3}$ as a single isomer. This experiment 
demonstrates that the chirality of the sulfinyl group is essential for achieving a good diastereoselection in this Mitsunobu cyclization.

Scheme 5. Preparation of trans-(2,2,3)-Trisubstituted Pyrrolidines Fully Stereocontrolled by the Chiral tert-Butylsulfinyl Group.
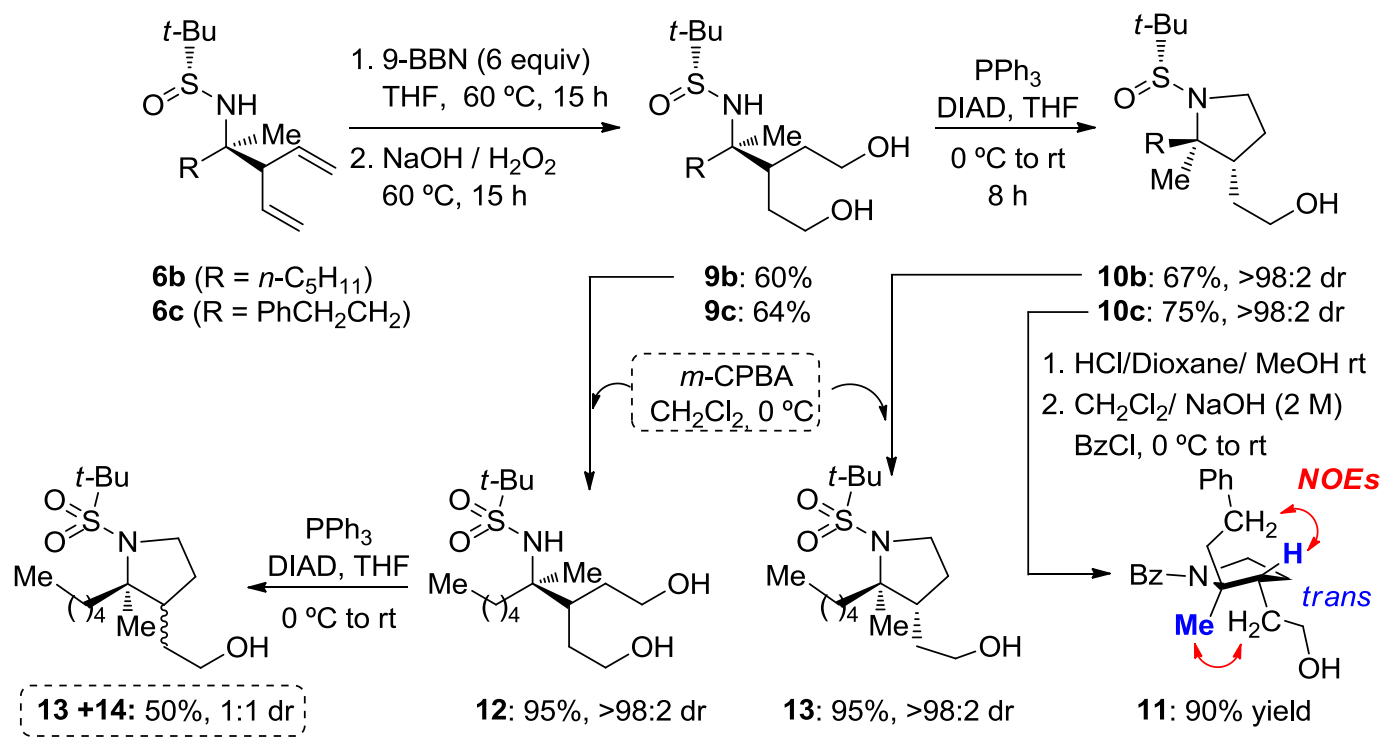

This excellent diastereoselectivity is noteworthy since both alkyl groups attached to the quaternary center exhibit similar steric bulkiness. Accordingly, we reasoned that this unexpected high diastereoselection should be supported on kinetic grounds. To account for the key role of the chiral sulfinyl group in the diastereoselection, we postulate two possible transition states where the oxygen of the sulfinyl group is hydrogen-bonded to the remained hydroxyethyl group. The pyrrolidine ring formation takes place from the transition state that avoids non-bonding interactions of the tert-butyl group with the substituents attached to C-2 (Scheme 6).

Scheme 6. Plausible Explanation for the Diastereoselective Mitsunobu Cyclization 

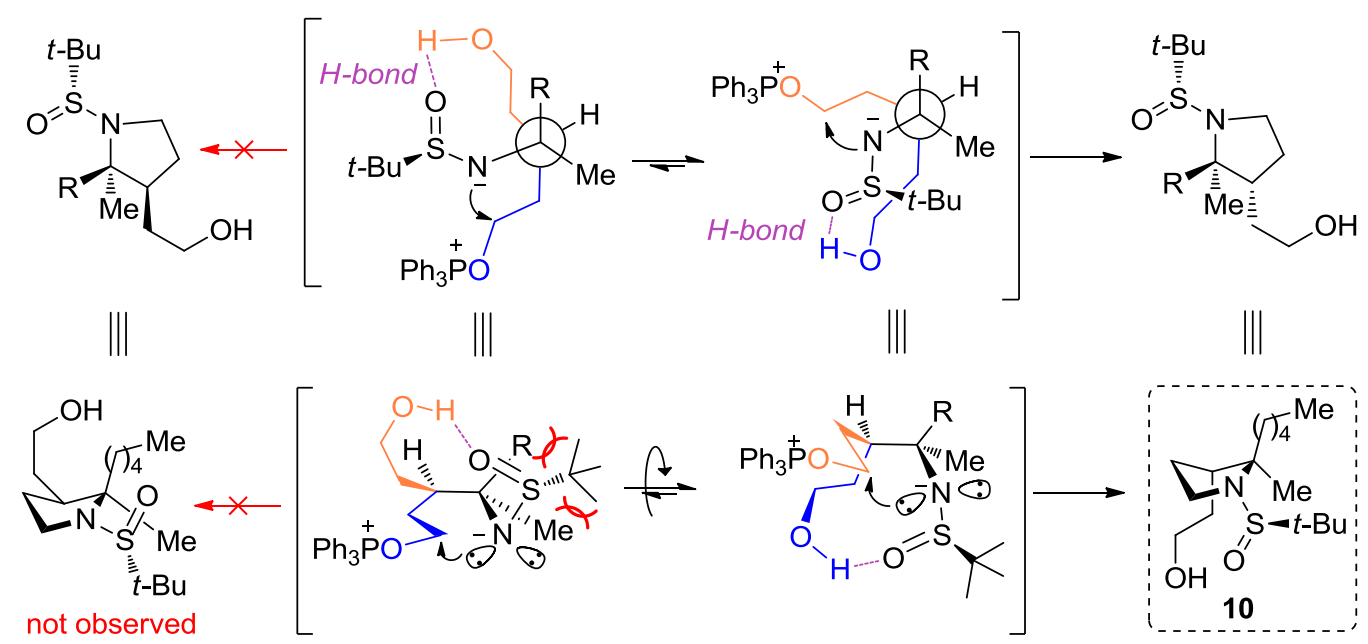

\section{CONCLUSION}

The aminopentadienylation of carbonyl compounds with chiral tert-butylsulfinamide and in situ-formed pentadienylindium reagent provides a convenient access to chiral $\alpha$ substituted amines with a 1,4-pentadien-3-yl unit from ready available starting materials. The protocol made use of $\operatorname{In}(0)$ and $\mathrm{Ti}(\mathrm{OEt})_{4}$, which are non-toxic and do not require a careful exclusion of moisture and/or air. This methodology accommodates electron-poor aromatic aldehydes, $\alpha, \beta$-unsaturated aldehydes, $\alpha$-branched aliphatic aldehydes and is particularly efficient -in terms of yields and diastereoselectivities- with $\alpha$-unsubstituted aldehydes and methylalkyl ketones. Catalytic hydrogenation of some of the pentadienyl amines obtained allowed the formation of enantioenriched $\alpha$-tertiary or quaternary-(3-pentyl)-amines, which are otherwise difficult to prepare. Moreover, the hydroboration-oxidation of selected examples of pentadienyl amines followed by a cyclization of the obtained amino diol under Mitsunobu reaction conditions, furnished the corresponding trans-2,2,3-trisubstituted pyrrolidines with excellent diastereoselectivity. It was demonstrated that the chirality of the sulfinyl group was essential for this high diastereoselection. 


\section{EXPERIMENTAL SECTION}

General Remarks. $\left(R_{\mathrm{s}}\right)-N$-tert-Butylsulfinyl amine $\mathbf{1}$ and its enantiomer (ent-1) were a gift of Medalchemy (> 99\% ee by chiral HPLC on a Chiracel AS column, $n$-Hexane/iPrOH 90:10, $1 \mathrm{~mL} / \mathrm{min}, \lambda=222 \mathrm{~nm})$. TLCs were performed on silica gel $60 \mathrm{~F}_{254}$, using aluminum plates and visualized with phosphomolybdic acid (PMA) or ninhydrin stain. Flash chromatography was carried out on handpacked columns of silica gel 60 (230400 mesh). Melting points are uncorrected. Optical rotations were measured using a polarimeter with a thermally jacketted $5 \mathrm{~cm}$ cell at approximately $20{ }^{\circ} \mathrm{C}$ and concentrations (c) are given in $\mathrm{g} / 100 \mathrm{~mL}$. Infrared analyses were performed with a spectrophotometer equipped with an ATR component; wavenumbers are given in $\mathrm{cm}^{-1}$. GC analyses were obtained with an HP-5 column $(30 \mathrm{~m} \times 0.25 \mathrm{~mm}$, i.d. $\times 0.25 \mu \mathrm{m})$ and an EI (70 EV) detector; the temperature program was as follows: hold at $60{ }^{\circ} \mathrm{C}$ for 3 min, ramp from 60 to $270{ }^{\circ} \mathrm{C}$ at $15^{\circ} \mathrm{C} / \mathrm{min}$, hold at $270{ }^{\circ} \mathrm{C}$ for $10 \mathrm{~min}$. Mass spectra (EI) were obtained at $70 \mathrm{EV}$; and fragment ions in $\mathrm{m} / \mathrm{z}$ with relative intensities (\%) in parentheses. HRMS analyses were also carried out in the electron impact mode (EI) at $70 \mathrm{eV}$ using a quadrupole mass analyzer or in the electrospray ionization mode (ESI) using a TOF analyzer. ${ }^{1} \mathrm{H}$ NMR spectra were recorded at 300 or $400 \mathrm{MHz}$, using $\mathrm{CDCl}_{3}$ or $\mathrm{CD}_{3} \mathrm{CN}$ as the solvent and TMS as an internal Standard $(0.00 \mathrm{ppm})$; the data is reported as $(\mathrm{s}=$ singlet, $\mathrm{d}=$ doublet, $\mathrm{t}=$ triplet, $\mathrm{m}=$ multiplet or unresolved, $\mathrm{br}=$ broad signal, coupling constant(s) in $\mathrm{Hz}$, integration). ${ }^{13} \mathrm{C}$ NMR spectra were recorded with ${ }^{1} \mathrm{H}$-decoupling at $101 \mathrm{MHz}$ using the solvent signal as reference (77.16 ppm for $\mathrm{CDCl}_{3}$ ). DEPT-135 experiments were performed to assign $\mathrm{CH}, \mathrm{CH}_{2}$ and $\mathrm{CH}_{3}$. 
2,4-pentadienyl bromide (3). ${ }^{25}$ To a stirring solution of $\mathrm{PBr}_{3}(190 \mu \mathrm{L}, 2 \mathrm{mmol})$ in dry $\mathrm{Et}_{2} \mathrm{O}(2.5 \mathrm{~mL})$ under an Ar atmosphere, was added 1,4-pentadien-3-ol (485 $\left.\mu \mathrm{L}, 5 \mathrm{mmol}\right)$ dropwise over ca. $2 \mathrm{~min}$ at $0{ }^{\circ} \mathrm{C}$. The resulting solution was stirred at $0{ }^{\circ} \mathrm{C}$ until the starting material disappeared (followed by GC, starting alcohol: $t_{R}=2.2$ min, product: $\left.t_{R}=4.0 \mathrm{~min}\right)$. The reaction was carefully quenched by the addition of brine $(1 \mathrm{~mL})$. The layers were separated and the organics were washed sequentially with a saturated solution of $\mathrm{NaHCO}_{3}(\mathrm{x} 3)$, brine, dried over $\mathrm{MgSO}_{4}$ and filtered. The volatiles were carefully removed at $40{ }^{\circ} \mathrm{C}$ under atmospheric pressure. The product was obtained as a colorless oil (447 mg, 60\%, 97wt\% in $\left.\mathrm{Et}_{2} \mathrm{O}\right):{ }^{1} \mathrm{H}$ NMR (300 MHz, $\left.\mathrm{CDCl}_{3}\right) \delta 6.46-6.22$ (m, 2H), $5.90(\mathrm{dt}, J=13.0,7.8 \mathrm{~Hz}, 1 \mathrm{H}), 5.28(\mathrm{~d}, J=14.9 \mathrm{~Hz}, 1 \mathrm{H}), 5.17(\mathrm{~d}, J=10.2 \mathrm{~Hz}$, $1 \mathrm{H}), 4.03(\mathrm{~d}, J=7.6 \mathrm{~Hz}, 2 \mathrm{H})$.

General procedure for the synthesis of sulfinamides 4. To a dry flask was added $\left(R_{\mathrm{S}}\right)$ $N$-tert-butylsulfinamide $(1,61 \mathrm{mg}, 0.5 \mathrm{mmol})$ followed by indium powder $(71 \mathrm{mg}, 0.63$ mmol). The reaction vessel was evacuated and put under an Ar atmosphere. Then a solution of the corresponding aldehyde $(0.55 \mathrm{mmol})$ in dry THF $(1 \mathrm{~mL})$ and $\mathrm{Ti}(\mathrm{OEt})_{4}$ (225 $\mu \mathrm{L}, 1 \mathrm{mmol})$ were added successively and the reaction mixture was stirred under an Ar for $1 \mathrm{~h}$ at $23{ }^{\circ} \mathrm{C}$. After this time, 2,4-pentadienyl bromide (110 mg, $\left.0.75 \mathrm{mmol}\right)$ was added to the mixture and it was heated to $60^{\circ} \mathrm{C}$ for $3 \mathrm{~h}$. The mixture was allowed to reach room temperature and was carefully added over a stirring mixture of 4:1 EtOAc/brine $(50 \mathrm{~mL})$. The resulting white suspension was filtered through a short pad of Celite, washed with EtOAc and the organics were concentrated under reduced pressure. The resulting suspension was diluted in 4:1 EtOAc/hexane $(50 \mathrm{~mL})$, filtered again through Celite and the organics were concentrated under reduced pressure.

$\left(R_{\mathbf{S}}, S\right)$-N-tert-Butylsulfinyl-1-phenyl-2-vinylbut-3-en-1-amine $\quad(4 a)$. The crude product was prepared from $\mathrm{PhCHO}$ following the general procedure and purified by 
column chromatography (7:3 hexane/EtOAc). The expected product was obtained as a yellow oil (19 mg, 14\%, 90:10 dr according to $\left.{ }^{1} \mathrm{H} \mathrm{NMR}\right)$ : $[\alpha]_{\mathrm{D}}{ }^{20}-121.5\left(c 1.3, \mathrm{CHCl}_{3}\right)$; $\mathrm{R}_{\mathrm{f}} 0.12\left(8: 2\right.$ hexane/EtOAc); IR $v 3280,3079,2958,1634,1455,1056,917 \mathrm{~cm}^{-1}$; for the major diastereoisomer: ${ }^{1} \mathrm{H}$ NMR $\left(300 \mathrm{MHz}, \mathrm{CDCl}_{3}\right) \delta 7.38-7.28(\mathrm{~m}, 5 \mathrm{H}), 5.81$ (ddd, $J=17.0,10.2,9.0 \mathrm{~Hz}, 1 \mathrm{H}), 5.57$ (ddd, $J=17.3,10.5,7.1 \mathrm{~Hz}, 1 \mathrm{H}), 5.33-5.20(\mathrm{~m}$, 2H), $5.03-4.88(\mathrm{~m}, 2 \mathrm{H}), 4.28(\mathrm{dd}, J=8.7,1.5 \mathrm{~Hz}, 1 \mathrm{H}), 3.92($ br s, $1 \mathrm{H}), 3.07(\mathrm{dd}, J=$ 16.2, 8.4 Hz, 1H), $1.18(\mathrm{~s}, 9 \mathrm{H}) ;{ }^{13} \mathrm{C}$ NMR (101 MHz, $\left.\mathrm{CDCl}_{3}\right) \delta 139.7(\mathrm{C}), 137.7(\mathrm{CH})$, $136.4(\mathrm{CH}), 128.9(\mathrm{CH}), 128.3(\mathrm{CH}), 127.9(\mathrm{CH}), 119.0\left(\mathrm{CH}_{2}\right), 117.5\left(\mathrm{CH}_{2}\right), 60.2(\mathrm{CH})$, $56.0(\mathrm{CH}), 55.8(\mathrm{C}), 22.8\left(\mathrm{CH}_{3}\right) ; \mathrm{CG} \mathrm{t} \mathrm{R}_{\mathrm{R}}=14.6 \mathrm{~min}$.; LRMS (EI) $\mathrm{m} / \mathrm{z}(\%) 154(13), 153$ (100), 137 (7), 136 (25), 129 (8), 105 (21), 104 (25), 77 (11); HRMS (EI) calcd for $\mathrm{C}_{16} \mathrm{H}_{23} \mathrm{NOS}-\mathrm{C}_{4} \mathrm{H}_{8} 221.0874$, found 221.0888.

$\left(R_{\mathrm{S}}, 1 S\right)$-N-tert-Butylsulfinyl-1-(4-chlorophenyl)-2-vinylbut-3-en-1-amine $(4 \mathrm{~b}) . \quad$ It was prepared from $p$-Chlorobenzaldehyde following the general procedure and purified by column chromatography (7:3 hexane/EtOAc). The expected product was obtained as a yellow oil (102 mg, 66\%, single diastereoisomer according to ${ }^{1} \mathrm{H}$ NMR): $[\alpha]_{\mathrm{D}}{ }^{20}$ 150.7 ( c 0.69, $\left.\mathrm{CHCl}_{3}\right) ; \mathrm{R}_{\mathrm{f}} 0.20$ (7:3 hexane/EtOAc); IR v 3277, 3080, 2979, 2959, 1737 , $1635,1597,1490,1062,1013,919,828 \mathrm{~cm}^{-1} ;{ }^{1} \mathrm{H}$ NMR $\left(300 \mathrm{MHz}, \mathrm{CDCl}_{3}\right) \delta 7.33-$ $7.27(\mathrm{~m}, 2 \mathrm{H}), 7.24-7.18(\mathrm{~m}, 2 \mathrm{H}), 5.78(\mathrm{ddd}, J=17.0,10.2,9.0 \mathrm{~Hz}, 1 \mathrm{H}), 5.54(\mathrm{ddd}, J=$ 17.4, 10.4, 7.2 Hz, 1H), 5.30 (dd, $J=10.3,1.6 \mathrm{~Hz}, 1 \mathrm{H}), 5.24$ (ddd, $J=17.1,1.6,0.7 \mathrm{~Hz}$, 1H), $5.00(\mathrm{dt}, J=10.4,1.3 \mathrm{~Hz}, 1 \mathrm{H}), 4.93(\mathrm{dt}, J=17.2,1.3 \mathrm{~Hz}, 1 \mathrm{H}), 4.26(\mathrm{dd}, J=8.6$, $1.3 \mathrm{~Hz}, 1 \mathrm{H}), 3.91(\mathrm{~s}, 1 \mathrm{H}), 3.02(\mathrm{dd}, J=16.4,8.6 \mathrm{~Hz}, 1 \mathrm{H}), 1.17(\mathrm{~s}, 9 \mathrm{H}) ;{ }^{13} \mathrm{C} \mathrm{NMR}(101$ $\left.\mathrm{MHz}, \mathrm{CDCl}_{3}\right) \delta 138.3(\mathrm{C}), 137.4(\mathrm{CH}), 136.1(\mathrm{CH}), 133.7(\mathrm{C}), 130.2(\mathrm{CH}), 128.6(\mathrm{CH})$, $119.3\left(\mathrm{CH}_{2}\right), 117.9\left(\mathrm{CH}_{2}\right), 59.5(\mathrm{CH}), 56.1(\mathrm{CH}), 55.9(\mathrm{C}), 22.7\left(\mathrm{CH}_{3}\right) ; \mathrm{CG} \mathrm{t}_{\mathrm{R}}=16.0$ min.; LRMS (EI) m/z (\%) 189 (33), 187 (100), 170 (5), 157 (3), 142 (5), 141 (14), 140 
(10), 139 (33), 138 (21), 128 (4), 67 (5); HRMS (ESI) calcd for $\mathrm{C}_{16} \mathrm{H}_{23} \mathrm{NOSCl}(\mathrm{M}+\mathrm{H})$ 312.1189, found 312.1185.

$\left(R_{\mathrm{S}}, 1 S\right)$-N-tert-Butylsulfinyl-1-(3-chlorophenyl)-2-vinylbut-3-en-1-amine (4c). It was prepared from $m$-Chlorobenzaldehyde following the general procedure and purified by column chromatography (7:3 hexane/EtOAc). The expected product was obtained as a colorless oil (129 mg, 83\%, 90:10 dr according to $\left.{ }^{1} \mathrm{H} \mathrm{NMR}\right):[\alpha]_{\mathrm{D}}{ }^{20}-105.8(c 0.72$, $\left.\mathrm{CHCl}_{3}\right) ; \mathrm{R}_{\mathrm{f}} 0.30$ (7:3 Hexane/EtOAc); IR v 3276, 3217, 2978, 2960, 1634, 1597, 1574, 1474, 1316, 1056, 920, $752 \mathrm{~cm}^{-1} ;{ }^{1} \mathrm{H}$ NMR (400 MHz, $\left.\mathrm{CDCl}_{3}\right) \delta 7.29-7.27(\mathrm{~m}, 1 \mathrm{H})$, $7.27-7.24(\mathrm{~m}, 2 \mathrm{H}), 7.19-7.14(\mathrm{~m}, 1 \mathrm{H}), 5.78(\mathrm{ddd}, J=17.1,10.2,9.0 \mathrm{~Hz}, 1 \mathrm{H}), 5.56$ (ddd, $J=17.4,10.4,7.2 \mathrm{~Hz}, 1 \mathrm{H}), 5.30(\mathrm{dd}, J=10.2,1.4 \mathrm{~Hz}, 1 \mathrm{H}), 5.25(\mathrm{dd}, J=17.1,0.7$ $\mathrm{Hz}, 1 \mathrm{H}), 5.04-4.99(\mathrm{~m}, 1 \mathrm{H}), 4.95(\mathrm{dt}, J=17.2,1.3 \mathrm{~Hz}, 1 \mathrm{H}), 4.26(\mathrm{dd}, J=8.6,1.2 \mathrm{~Hz}$ 1H), $3.91(\mathrm{~s}, 1 \mathrm{H}), 3.03(\mathrm{q}, J=8.4 \mathrm{~Hz}, 1 \mathrm{H}), 1.19(\mathrm{~s}, 9 \mathrm{H}) ;{ }^{13} \mathrm{C} \mathrm{NMR}\left(101 \mathrm{MHz}, \mathrm{CDCl}_{3}\right) \delta$ $142.0(\mathrm{C}), 137.3(\mathrm{CH}), 136.0(\mathrm{CH}), 134.3(\mathrm{C}), 129.5(\mathrm{CH}), 128.7(\mathrm{CH}), 128.1(\mathrm{CH})$, $127.2(\mathrm{CH}), 119.4\left(\mathrm{CH}_{2}\right), 118.0\left(\mathrm{CH}_{2}\right), 59.7(\mathrm{CH}), 56.0(\mathrm{CH}), 55.9(\mathrm{C}), 22.7\left(\mathrm{CH}_{3}\right) ; \mathrm{CG}$ $\mathrm{t}_{\mathrm{R}}=15.8$ min.; LRMS (EI) m/z (\%) 189 (38), 188 (10), 187 (100), 170 (12), 157 (4), 142 (6), 141 (13), 140 (10), 139 (28), 138 (20), 128 (5), 67 (5); HRMS (ESI) calcd for $\mathrm{C}_{16} \mathrm{H}_{23} \mathrm{NOSCl} 312.1189$, found 312.1186.

$\left(R_{S}, 1 S\right)$-N-tert-Butylsulfinyl-1-(2-chlorophenyl)-2-vinylbut-3-en-1-amine $\quad(4 d) . \quad$ It was prepared from $o$-Chlorobenzaldehyde following the general procedure and purified by column chromatography (7:3 hexane/EtOAc). The expected product was obtained as a colorless oil (33 mg, 21\%, single diastereoisomer according to ${ }^{1} \mathrm{H}$ NMR): $[\alpha]_{\mathrm{D}}{ }^{20}{ }_{-}$ 113.8 ( $c$ 0.60, $\left.\mathrm{CHCl}_{3}\right) ; \mathrm{R}_{\mathrm{f}} 0.29$ (7:3 hexane/EtOAc); IR v 3281, 3079, 2978, 2959, 1634, 1573, 1473, 1363, 1062, 919, $730 \mathrm{~cm}^{-1} ;{ }^{1} \mathrm{H}$ NMR (300 MHz, $\left.\mathrm{CDCl}_{3}\right) \delta 7.38-7.32(\mathrm{~m}$, 2H), $7.25(\mathrm{td}, J=7.5,1.5 \mathrm{~Hz}, 1 \mathrm{H}), 7.22-7.17(\mathrm{~m}, 1 \mathrm{H}), 5.84(\mathrm{ddd}, J=17.1,10.2,8.7$ $\mathrm{Hz}, 1 \mathrm{H}), 5.74(\mathrm{ddd}, J=17.4,10.4,7.3 \mathrm{~Hz}, 1 \mathrm{H}), 5.28(\mathrm{dd}, J=10.2,1.4 \mathrm{~Hz}, 1 \mathrm{H}), 5.21(\mathrm{~d}$, 
$J=17.1 \mathrm{~Hz}, 1 \mathrm{H}), 5.02(\mathrm{~d}, J=10.4 \mathrm{~Hz}, 1 \mathrm{H}), 4.97(\mathrm{dt}, J=17.1,1.4 \mathrm{~Hz}, 1 \mathrm{H}), 4.94(\mathrm{~s}, 1 \mathrm{H})$, $3.86(\mathrm{~s}, 1 \mathrm{H}), 3.18(\mathrm{dd}, J=15.1,7.4 \mathrm{~Hz}, 1 \mathrm{H}), 1.17(\mathrm{~s}, 9 \mathrm{H}) ;{ }^{13} \mathrm{C} \mathrm{NMR}\left(101 \mathrm{MHz}, \mathrm{CDCl}_{3}\right)$ $\delta 137.8(\mathrm{C}), 136.9(\mathrm{CH}), 136.1(\mathrm{CH}), 134.4(\mathrm{C}), 129.8(\mathrm{CH}), 129.7(\mathrm{CH}), 128.7(\mathrm{CH})$, $126.7(\mathrm{CH}), 119.3\left(\mathrm{CH}_{2}\right), 117.7\left(\mathrm{CH}_{2}\right), 56.1(\mathrm{CH}), 55.9(\mathrm{C}), 55.1(\mathrm{CH}), 22.7\left(\mathrm{CH}_{3}\right) ; \mathrm{CG}$ $\mathrm{t}_{\mathrm{R}}=15.5$ min.; LRMS (EI) $\mathrm{m} / z(\%) 189$ (30), 187 (100), 170 (7), $142(6), 141(13), 140$ (10), 139 (30), 138 (21), 128 (6), 67 (5); HRMS (ESI) calcd for $\mathrm{C}_{16} \mathrm{H}_{23} \mathrm{NOSCl}$ 312.1189, found 312.1187.

$\left(R_{\mathrm{S}}, 1 R\right)$-N-tert-Butylsulfinyl-1-cyclohexyl-2-vinylbut-3-en-1-amine (4e). The crude product prepared from cyclohexanecarbaldehyde was obtained as a mixture of $\alpha$ - and $\gamma$ allylic products (26:74 according ${ }^{1} \mathrm{H}$ NMR) following the general procedure. The desired $\gamma$ - product was purified by column chromatography (9:1 hexane/ EtOAc) giving a colorless wax (78 mg, 55\%, single diastereoisomer according to $\left.{ }^{1} \mathrm{H} \mathrm{NMR}\right):[\alpha]_{\mathrm{D}}{ }^{20}-$ 72.8 ( $c$ 0.73, $\left.\mathrm{CHCl}_{3}\right) ; \mathrm{R}_{\mathrm{f}} 0.20$ (8:2 hexane/EtOAc); IR v 3292, 3232, 3075, 2978, 2924, 2852, 1638, 1449, 1363, 1059, 995, 912, $752 \mathrm{~cm}^{-1} ;{ }^{1} \mathrm{H}$ NMR $\left(300 \mathrm{MHz}, \mathrm{CDCl}_{3}\right) \delta 5.94$ $-5.81(\mathrm{~m}, 2 \mathrm{H}), 5.21-5.07(\mathrm{~m}, 4 \mathrm{H}), 3.32(\mathrm{~d}, J=5.3 \mathrm{~Hz}, 1 \mathrm{H}), 3.15-3.05(\mathrm{~m}, 2 \mathrm{H}), 1.79$ $-1.48(\mathrm{~m}, 6 \mathrm{H}), 1.23(\mathrm{~s}, 9 \mathrm{H}), 1.21-0.97(\mathrm{~m}, 5 \mathrm{H}) .{ }^{13} \mathrm{C} \mathrm{NMR}\left(101 \mathrm{MHz}, \mathrm{CDCl}_{3}\right) \delta 138.6$ $(\mathrm{CH}), 138.1(\mathrm{CH}), 117.4\left(\mathrm{CH}_{2}\right), 117.3\left(\mathrm{CH}_{2}\right), 62.7(\mathrm{CH}), 56.5(\mathrm{C}), 52.0(\mathrm{CH}), 40.7$ $(\mathrm{CH}), 31.5\left(\mathrm{CH}_{2}\right), 27.8\left(\mathrm{CH}_{2}\right), 26.7\left(\mathrm{CH}_{2}\right), 26.6\left(\mathrm{CH}_{2}\right), 26.3\left(\mathrm{CH}_{2}\right), 23.1\left(\mathrm{CH}_{3}\right) ; \mathrm{CG} \mathrm{t}_{\mathrm{R}}=$ 14.7 min.; LRMS (EI) m/z (\%) 227 (7), 160 (24), 159 (100), 144 (59), 96 (31), 95 (53), 94 (11), 81 (32), 79 (11), 77 (28), 68 (13), 67 (22), 55 (18); HRMS (EI) calcd for $\mathrm{C}_{16} \mathrm{H}_{29} \mathrm{NOS}-\mathrm{C}_{4} \mathrm{H}_{8} 227.1344$, found 227.1339.

$\left(\boldsymbol{R}_{\mathbf{S}}, \mathbf{4 R}\right)$ - $\boldsymbol{N}$-tert-Butylsulfinyl-3-vinyltridec-1-en-4-amine (4f). The crude product (93:7 dr according ${ }^{1} \mathrm{H}$ NMR) prepared from decanal following the general procedure was purified by column chromatography (9:1 hexane/EtOAc). The expected product was obtained as a yellow oil (150 mg, 90\%, 98:2 dr according to ${ }^{1} \mathrm{H}$ NMR): $[\alpha]_{\mathrm{D}}{ }^{20}-50.3(c$ 
1.01, $\left.\mathrm{CHCl}_{3}\right) ; \mathrm{R}_{\mathrm{f}} 0.23$ (8:2 hexane/EtOAc); IR v 3290, 3209, 3077, 2954, 2924, 2854, 1635, 1466, 1362, 1065, 999, 914, $721 \mathrm{~cm}^{-1} ;{ }^{1} \mathrm{H} \mathrm{NMR}\left(300 \mathrm{MHz}, \mathrm{CDCl}_{3}\right) \delta 5.86-5.80$ (m, 1H), $5.80-5.74(\mathrm{~m}, 1 \mathrm{H}), 5.27-5.07(\mathrm{~m}, 4 \mathrm{H}), 3.42(\mathrm{~d}, J=7.0 \mathrm{~Hz}, 1 \mathrm{H}), 3.32-3.23$ $(\mathrm{m}, 1 \mathrm{H}), 3.16(\mathrm{dd}, J=13.5,7.3 \mathrm{~Hz}, 1 \mathrm{H}), 1.56-1.50(\mathrm{~m}, 1 \mathrm{H}), 1.32-1.23(\mathrm{~m}, 15 \mathrm{H})$, $1.21(\mathrm{~s}, 9 \mathrm{H}), 0.88(\mathrm{t}, J=6.9 \mathrm{~Hz}, 3 \mathrm{H}) ;{ }^{13} \mathrm{C} \mathrm{NMR}\left(101 \mathrm{MHz}, \mathrm{CDCl}_{3}\right) \delta 137.8(\mathrm{CH}), 136.4$ $(\mathrm{CH}), 119.1\left(\mathrm{CH}_{2}\right), 117.2\left(\mathrm{CH}_{2}\right), 58.8(\mathrm{CH}), 56.2(\mathrm{C}), 53.3(\mathrm{CH}), 32.0\left(\mathrm{CH}_{2}\right), 31.5$ $\left(\mathrm{CH}_{2}\right), 29.7\left(\mathrm{CH}_{2}\right), 29.7\left(\mathrm{CH}_{2}\right), 29.5\left(\mathrm{CH}_{2}\right), 29.4\left(\mathrm{CH}_{2}\right), 25.6\left(\mathrm{CH}_{2}\right), 22.9\left(\mathrm{CH}_{3}\right), 22.8$ $\left(\mathrm{CH}_{2}\right), 14.3\left(\mathrm{CH}_{3}\right) ; \mathrm{CG} \mathrm{t}_{\mathrm{R}}=16.3$ min.; LRMS (EI) $\mathrm{m} / z$ (\%) 271 (7), 270 (1), 222 (11), 204 (16), 203 (100), 156 (7), 95 (14), 84 (30), 70 (48), 55 (20); HRMS (EI) calcd for $\mathrm{C}_{19} \mathrm{H}_{37} \mathrm{NOS}-\mathrm{C}_{4} \mathrm{H}_{8} 271.1970$, found 271.1973.

$\left(\boldsymbol{R}_{\mathrm{S}}, \mathbf{4 R}\right)$-N-tert-Butylsulfinyl-8-bromo-3-vinyloct-1-en-4-amine $\quad(4 \mathrm{~g}) . \quad$ The crude product (94:6 dr according to ${ }^{1} \mathrm{H}$ NMR) prepared from 5-bromopentanal ${ }^{26}$ following the general procedure was purified by column chromatography (8:2 Hexane/EtOAc). The expected product was obtained as a yellow oil (122 mg, 73\%, single diastereoisomer according to ${ }^{1} \mathrm{H}$ NMR): $[\alpha]_{\mathrm{D}}{ }^{20}-52.4\left(\right.$ c 1.13, $\left.\mathrm{CHCl}_{3}\right) ; \mathrm{R}_{\mathrm{f}} 0.27$ (7:3 Hexane/EtOAc); ${ }^{1} \mathrm{H}$ NMR (300 MHz, $\left.\mathrm{CDCl}_{3}\right) \delta 5.87-5.69(\mathrm{~m}, 2 \mathrm{H}), 5.36-5.05(\mathrm{~m}, 4 \mathrm{H}), 3.45(\mathrm{dd}, J=6.8$, $5.1 \mathrm{~Hz}, 1 \mathrm{H}), 3.40(\mathrm{t}, J=6.6 \mathrm{~Hz}, 2 \mathrm{H}), 3.32-3.22(\mathrm{~m}, 1 \mathrm{H}), 3.16(\mathrm{dd}, J=14.2,6.9 \mathrm{~Hz}$, $1 \mathrm{H}), 1.96-1.76(\mathrm{~m}, 2 \mathrm{H}), 1.68-1.54(\mathrm{~m}, 2 \mathrm{H}), 1.49-1.29(\mathrm{~m}, 2 \mathrm{H}), 1.22(\mathrm{~s}, 9 \mathrm{H}) ;{ }^{13} \mathrm{C}$ NMR (101 MHz, $\left.\mathrm{CDCl}_{3}\right) \delta 137.6(\mathrm{CH}), 136.1(\mathrm{CH}), 119.4\left(\mathrm{CH}_{2}\right), 117.5\left(\mathrm{CH}_{2}\right), 58.4$ $(\mathrm{CH}), 56.2(\mathrm{C}), 53.2(\mathrm{CH}), 33.8\left(\mathrm{CH}_{2}\right), 32.6\left(\mathrm{CH}_{2}\right), 30.6\left(\mathrm{CH}_{2}\right), 24.2\left(\mathrm{CH}_{2}\right), 22.9\left(\mathrm{CH}_{3}\right)$; $\mathrm{CG} \mathrm{t}_{\mathrm{R}}=15.4$ min.; LRMS (EI) m/z (\%) 281 (4), 279 (4), 214 (9), 213 (100), 212 (10), 211 (97), 200 (7), 144 (10), 104 (8), 95 (11), 85 (5), 84 (38), 83 (4), 81 (22), 79 (9), 77 (17), 69 (14), 68 (24), 67 (45), 56 (12), 55 (18), 53 (12); HRMS (EI) calcd for $\mathrm{C}_{14} \mathrm{H}_{26} \mathrm{BrNOS}-\mathrm{C}_{4} \mathrm{H}_{8}$ 279.0292, found 279.0290. 
$\left(R_{\mathrm{S}}, 2 R\right)$ - $N$-tert-Butylsulfinyl-1-phenyl-3-vinylpent-4-en-2-amine $(4 \mathrm{~h})$. The crude product (97:3 dr according ${ }^{1} \mathrm{H}$ NMR) was prepared from phenylethanal, following the general procedure, and purified by column chromatography (8:2 Hexane/EtOAc). The expected product was obtained as a yellow oil (102 mg, 70\%, 98:2 dr according to ${ }^{1} \mathrm{H}$ NMR): $[\alpha]_{\mathrm{D}}^{20}-21.1\left(c 0.73, \mathrm{CHCl}_{3}\right) ; \mathrm{R}_{\mathrm{f}} 0.29$ (7:3 Hexane/EtOAc); IR v 3291, 3074, 2981, 1495, 1455, 1216, 1057, 921, $747 \mathrm{~cm}^{-1} ;{ }^{1} \mathrm{H}$ NMR $\left(300 \mathrm{MHz}, \mathrm{CDCl}_{3}\right) \delta 7.31-$ $7.13(\mathrm{~m}, 5 \mathrm{H}), 5.99-5.79(\mathrm{~m}, 2 \mathrm{H}), 5.36-5.12(\mathrm{~m}, 4 \mathrm{H}), 3.59(\mathrm{ddd}, J=13.6,8.2,4.7 \mathrm{~Hz}$, 1H), $3.44(\mathrm{~d}, J=7.2 \mathrm{~Hz}, 1 \mathrm{H}), 3.22(\mathrm{dd}, J=12.2,7.3 \mathrm{~Hz}, 1 \mathrm{H}), 2.91(\mathrm{dd}, J=14.0,4.9$ $\mathrm{Hz}, 1 \mathrm{H}), 2.60(\mathrm{dd}, J=13.9,9.0 \mathrm{~Hz}, 1 \mathrm{H}), 1.04(\mathrm{~s}, 9 \mathrm{H}) ;{ }^{13} \mathrm{C} \mathrm{NMR}\left(101 \mathrm{MHz}, \mathrm{CDCl}_{3}\right) \delta$ $138.9(\mathrm{C}), 136.9(\mathrm{CH}), 136.2(\mathrm{CH}), 129.5(\mathrm{CH}), 128.2(\mathrm{CH}), 126.2(\mathrm{CH}), 119.5\left(\mathrm{CH}_{2}\right)$, $117.8\left(\mathrm{CH}_{2}\right), 60.8(\mathrm{CH}), 56.0(\mathrm{C}), 52.4(\mathrm{CH}), 38.2\left(\mathrm{CH}_{2}\right), 22.5\left(\mathrm{CH}_{3}\right) ; \mathrm{CG} \mathrm{t}_{\mathrm{R}}=16.4$ min.; LRMS (EI) m/z (\%) 235 (5), 167 (5), 146 (5), 145 (8), 144 (100), 128 (6), 104 (24), 92 (7), 91 (35), 81 (19), 68 (4); HRMS (EI) calcd for $\mathrm{C}_{17} \mathrm{H}_{25} \mathrm{NOS}-\mathrm{C}_{4} \mathrm{H}_{8}$ 235.1031, found 235.1032.

$\left(S_{\mathrm{S}}, 2 S\right)$ - $N$-tert-Butylsulfinyl-1-phenyl-3-vinylpent-4-en-2-amine $\quad(e n t-4 h) . \quad$ It was prepared from $\left(S_{\mathrm{S}}\right)$-N-tert-butylsulfinamide (ent-1) following the same general procedure obtaining a yellow oil (100 mg, 69\%). Physical and spectroscopy data were found to be the same than for $\mathbf{4 h}$, except for the optical rotation: $[\alpha]_{D}{ }^{20}+20.4(c 1.2$, $\left.\mathrm{CHCl}_{3}\right)$.

$\left(\boldsymbol{R}_{\mathrm{S}}, 3 R\right)-N$-tert-Butylsulfinyl-1-phenyl-4-vinylhex-5-en-3-amine (4i). Compound $4 \mathrm{i}$ was prepared from 3-phenylpropanal following the general procedure. After purification by column chromatography (8:2 Hexane/EtOAc), the expected product was obtained as a yellow oil $\left(130 \mathrm{mg}, 85 \%, 97: 3 \mathrm{dr}\right.$ according to $\left.{ }^{1} \mathrm{H} \mathrm{NMR}\right)$ : $[\alpha]_{\mathrm{D}}{ }^{20}-61.5(c 0.85$, $\left.\mathrm{CHCl}_{3}\right) ; \mathrm{R}_{\mathrm{f}} 0.17$ (8:2 Hexane/EtOAc); IR v 3286, 3079, 2977, 2950, 1635, 1602, 1455 , 1057, $917 \mathrm{~cm}^{-1} ;{ }^{1} \mathrm{H}$ NMR $\left(300 \mathrm{MHz}, \mathrm{CDCl}_{3}\right) \delta 7.34-7.29(\mathrm{~m}, 2 \mathrm{H}), 7.27-7.14(\mathrm{~m}$, 
$3 \mathrm{H}), 5.88-5.71(\mathrm{~m}, 2 \mathrm{H}), 5.34-5.23(\mathrm{~m}, 2 \mathrm{H}), 5.23-5.11(\mathrm{~m}, 2 \mathrm{H}), 3.56(\mathrm{~d}, J=7.1 \mathrm{~Hz}$, $1 \mathrm{H}), 3.35(\mathrm{tdd}, J=7.2,5.3,3.6 \mathrm{~Hz}, 1 \mathrm{H}), 3.24(\mathrm{dd}, J=13.9,6.9 \mathrm{~Hz}, 1 \mathrm{H}), 2.82-2.73(\mathrm{~m}$, 1H), $2.61(\mathrm{ddd}, J=13.7,10.4,6.3 \mathrm{~Hz}, 1 \mathrm{H}), 1.99-1.89(\mathrm{~m}, 1 \mathrm{H}), 1.72-1.60(\mathrm{~m}, 1 \mathrm{H})$, $1.28(\mathrm{~s}, 9 \mathrm{H}) ;{ }^{13} \mathrm{C}$ NMR (101 MHz, $\left.\mathrm{CDCl}_{3}\right) \delta 142.0(\mathrm{C}), 137.7(\mathrm{CH}), 136.0(\mathrm{CH}), 128.6$ $(\mathrm{CH}), 128.5(\mathrm{CH}), 126.1(\mathrm{CH}), 119.6\left(\mathrm{CH}_{2}\right), 117.4\left(\mathrm{CH}_{2}\right), 58.3(\mathrm{CH}), 56.3(\mathrm{C}), 53.3$ $(\mathrm{CH}), 33.6\left(\mathrm{CH}_{2}\right), 32.0\left(\mathrm{CH}_{2}\right), 23.0\left(\mathrm{CH}_{3}\right), ; \mathrm{CG} \mathrm{t} \mathrm{R}=16.3 \mathrm{~min} . ; \mathrm{LRMS}(\mathrm{EI}) \mathrm{m} / z(\%) 249$ $\left(\mathrm{M}^{+}-\mathrm{C}_{4} \mathrm{H}_{8}, 8\right), 181$ (18), 145 (8), 133 (11), 132 (10), 118 (12), 117 (81), 96 (24), 92 (10), 91 (100), 81 (5), 77 (8), 67 (9), 65 (10); HRMS (EI) calcd for $\mathrm{C}_{18} \mathrm{H}_{27} \mathrm{NOS}-\mathrm{C}_{4} \mathrm{H}_{8}$ 249.1187, found 249.1176.

$\left(S_{\mathrm{S}}, 3 S\right)$-N-tert-Butylsulfinyl-1-phenyl-4-vinylhex-5-en-3-amine $\quad(e n t-4 \mathrm{i}) . \quad$ It $\quad$ was prepared from $\left(S_{\mathrm{S}}\right)-\mathrm{N}$-tert-butylsulfinamide (ent-1) following the same general procedure, obtaining a colorless oil (122 mg, 80\%, 97:3 dr according to ${ }^{1} \mathrm{H}$ NMR). Physical and spectroscopy data were found to be the same than for $\mathbf{4 i}$, except for the optical rotation: $[\alpha]_{\mathrm{D}}^{20}+56.4\left(c 1.7, \mathrm{CHCl}_{3}\right)$.

$\left(R_{\mathrm{S}}, 1 E, 3 R\right)$ - $N$-tert-Butylsulfinyl-1-phenyl-4-vinylhexa-1,5-dien-3-amine

$(4 \mathbf{j})$.

Compound $\mathbf{4} \mathbf{j}$ was prepared from cinnamaldehyde following the general procedure. After purification by column chromatography (9:1 Hexane/EtOAc), the expected product was obtained as a white solid (109 $\mathrm{mg}, 72 \%, 97: 3 \mathrm{dr}$ according to ${ }^{1} \mathrm{H}$ NMR): mp $47.9-50.0{ }^{\circ} \mathrm{C} ;[\alpha]_{\mathrm{D}}{ }^{20}+133.5\left(c 1.01, \mathrm{CHCl}_{3}\right) ; \mathrm{R}_{\mathrm{f}} 0.30$ (7:3 Hexane/EtOAc); IR v $3281,3079,2977,1635,1363,1059,966,918,752 \mathrm{~cm}^{-1} ;{ }^{1} \mathrm{H} \mathrm{NMR}\left(300 \mathrm{MHz}, \mathrm{CDCl}_{3}\right) \delta$ $7.42-7.28(\mathrm{~m}, 4 \mathrm{H}), 7.27-7.22(\mathrm{~m}, 1 \mathrm{H}), 6.61(\mathrm{~d}, J=15.8 \mathrm{~Hz}, 1 \mathrm{H}), 5.98(\mathrm{dd}, J=15.9$, $8.0 \mathrm{~Hz}, 1 \mathrm{H}), 5.95-5.75(\mathrm{~m}, 2 \mathrm{H}), 5.32-5.12(\mathrm{~m}, 4 \mathrm{H}), 3.99(\mathrm{td}, J=7.2,2.6 \mathrm{~Hz}, 1 \mathrm{H})$, $3.67(\mathrm{~d}, J=2.7 \mathrm{~Hz}, 1 \mathrm{H}), 3.05(\mathrm{q}, J=7.3 \mathrm{~Hz}, 1 \mathrm{H}), 1.23(\mathrm{~s}, 9 \mathrm{H}) ;{ }^{13} \mathrm{C} \mathrm{NMR}(101 \mathrm{MHz}$, $\left.\mathrm{CDCl}_{3}\right) \delta 137.2(\mathrm{CH}), 136.7(\mathrm{C}), 136.5(\mathrm{CH}), 133.9(\mathrm{CH}), 128.7(\mathrm{CH}), 128.0(\mathrm{CH})$, $127.9(\mathrm{CH}), 126.7(\mathrm{CH}), 118.5\left(\mathrm{CH}_{2}\right), 118.3\left(\mathrm{CH}_{2}\right), 59.1(\mathrm{CH}), 55.8(\mathrm{C}), 54.3(\mathrm{CH})$, 
$22.8\left(\mathrm{CH}_{3}\right) ; \mathrm{CG} \mathrm{t}_{\mathrm{R}}=16.7$ min.; LRMS (EI) m/z (\%) 228 (5), 181 (6), 180 (11), 179 (97), 162 (5), 141 (5), 131 (13), 130 (100), 129 (8), 117 (12), 116 (88), 115 (39), 103 (11), 91 (14), 78 (5), 77 (17), 67 (6); HRMS (EI) calcd for $\mathrm{C}_{18} \mathrm{H}_{25} \mathrm{NOS}-\mathrm{C}_{4} \mathrm{H}_{8}$ 247.1031, found 247.1040.

$\left(R_{S}, 4 R, 6 S\right)$-N-tert-Butylsulfinyl-6,10-dimethyl-3-vinylundeca-1,9-dien-4-amine (4k).

The product was prepared from $(S)$-citronellal, following the general procedure, and purified by column chromatography (8:2 Hexane/EtOAc). The expected product was obtained as a yellow oil $\left(139 \mathrm{mg}, 86 \%,>97: 3 \mathrm{dr}\right.$ according to $\left.{ }^{13} \mathrm{C} \mathrm{NMR}\right):[\alpha]_{\mathrm{D}}{ }^{20}-31.5$ (c 0.93, $\left.\mathrm{CHCl}_{3}\right) ; \mathrm{R}_{\mathrm{f}} 0.32$ (7:3 Hexane/EtOAc); IR $v$ 3288, 3080, 2958, 2928, 1635, 1457, 1363, 1059, 1001, 917, $798 \mathrm{~cm}^{-1} ;{ }^{1} \mathrm{H}$ NMR (300 MHz, $\left.\mathrm{CDCl}_{3}\right) \delta 5.90-5.70(\mathrm{~m}$, $2 \mathrm{H}), 5.31-5.20(\mathrm{~m}, 2 \mathrm{H}), 5.15(\mathrm{dt}, J=4.5,1.6 \mathrm{~Hz}, 1 \mathrm{H}), 5.11(\mathrm{dt}, J=11.0,1.6 \mathrm{~Hz}, 1 \mathrm{H})$, $5.08-5.02(\mathrm{~m}, 1 \mathrm{H}), 3.44-3.31(\mathrm{~m}, 2 \mathrm{H}), 3.26(\mathrm{t}, J=6.9 \mathrm{~Hz}, 1 \mathrm{H}), 1.96(\mathrm{q}, J=7.3 \mathrm{~Hz}$, 2H), $1.66(\mathrm{~d}, J=1.0 \mathrm{~Hz}, 3 \mathrm{H}), 1.59(\mathrm{~s}, 3 \mathrm{H}), 1.57-1.52(\mathrm{~m}, 1 \mathrm{H}), 1.35-1.23(\mathrm{~m}, 4 \mathrm{H})$, $1.21(\mathrm{~s}, 9 \mathrm{H}), 0.85(\mathrm{~d}, J=6.6 \mathrm{~Hz}, 3 \mathrm{H}) ;{ }^{13} \mathrm{C} \mathrm{NMR}\left(101 \mathrm{MHz}, \mathrm{CDCl}_{3}\right) \delta 137.8(\mathrm{CH}), 135.7$ $(\mathrm{CH}), 131.2(\mathrm{C}), 124.7(\mathrm{CH}), 119.8\left(\mathrm{CH}_{2}\right), 116.7\left(\mathrm{CH}_{2}\right), 57.9(\mathrm{CH}), 56.2(\mathrm{C}), 53.9(\mathrm{CH})$, $38.9\left(\mathrm{CH}_{2}\right), 37.8\left(\mathrm{CH}_{2}\right), 28.5(\mathrm{CH}), 25.7\left(\mathrm{CH}_{3}\right), 25.4\left(\mathrm{CH}_{2}\right), 22.7\left(\mathrm{CH}_{3}\right), 18.7\left(\mathrm{CH}_{3}\right)$, $17.7\left(\mathrm{CH}_{3}\right) ; \mathrm{CG} \mathrm{t}_{\mathrm{R}}=15.5$ min.; LRMS (EI) $\mathrm{m} / z$ (\%) 220 (33), 201 (12), 193 (23), 178 (45), 168 (5), 152 (76), 137 (49), 121 (38), 109 (100), 96 (44), 81 (97), 69 (89), 55 (35); HRMS (EI) calcd for $\mathrm{C}_{19} \mathrm{H}_{35} \mathrm{NOS}-\mathrm{C}_{4} \mathrm{H}_{8} 269.1813$, found 269.1808.

$\left(S_{\mathrm{S}}, 4 S, 6 S\right)$ - $N$-tert-Butylsulfinyl-6,10-dimethyl-3-vinylundeca-1,9-dien-4-amine (4I). The product was prepared from $(S)$-citronellal and ent-1, following the general procedure, and purified by column chromatography (8:2 Hexane/EtOAc). The expected product was obtained as a yellow oil $\left(137 \mathrm{mg}, 84 \%\right.$, >97:3 $\mathrm{dr}$ according to $\left.{ }^{13} \mathrm{C} \mathrm{NMR}\right)$ : $[\alpha]_{\mathrm{D}}{ }^{20}+30.5\left(c\right.$ 1.02, $\left.\mathrm{CHCl}_{3}\right) ; \mathrm{R}_{\mathrm{f}} 0.28(7: 3$ Hexane/EtOAc); IR $v$ 3290, 3077, 2958, 2924, 1635, 1456, 1362, 1059, 1001, 916, $794 \mathrm{~cm}^{-1} ;{ }^{1} \mathrm{H}$ NMR $\left(300 \mathrm{MHz}, \mathrm{CDCl}_{3}\right) \delta 5.87$ 
$-5.74(\mathrm{~m}, 2 \mathrm{H}), 5.32-5.21(\mathrm{~m}, 2 \mathrm{H}), 5.18-5.05(\mathrm{~m}, 3 \mathrm{H}), 3.43-3.34(\mathrm{~m}, 2 \mathrm{H}), 3.24(\mathrm{t}, J$ $=8.2 \mathrm{~Hz}, 1 \mathrm{H}), 2.00(\mathrm{td}, J=15.0,6.8 \mathrm{~Hz}, 1 \mathrm{H}), 1.94-1.83(\mathrm{~m}, 1 \mathrm{H}), 1.68(\mathrm{~d}, J=1.0 \mathrm{~Hz}$, 3H), $1.60(\mathrm{~s}, 3 \mathrm{H}), 1.45-1.35(\mathrm{~m}, 3 \mathrm{H}), 1.21(\mathrm{~s}, 9 \mathrm{H}), 1.19-1.13(\mathrm{~m}, 1 \mathrm{H}), 1.13-1.01(\mathrm{~m}$, $1 \mathrm{H}), 0.89(\mathrm{~d}, J=6.7 \mathrm{~Hz}, 3 \mathrm{H}) ;{ }^{13} \mathrm{C} \mathrm{NMR}\left(101 \mathrm{MHz}, \mathrm{CDCl}_{3}\right) \delta 137.4(\mathrm{CH}), 136.0(\mathrm{CH})$, $131.2(\mathrm{C}), 124.7(\mathrm{CH}), 119.6\left(\mathrm{CH}_{2}\right), 117.0\left(\mathrm{CH}_{2}\right), 57.6(\mathrm{CH}), 56.1(\mathrm{C}), 53.3(\mathrm{CH}), 39.3$ $\left(\mathrm{CH}_{2}\right), 35.6\left(\mathrm{CH}_{2}\right), 28.7(\mathrm{CH}), 25.7\left(\mathrm{CH}_{3}\right), 25.1\left(\mathrm{CH}_{2}\right), 22.7\left(\mathrm{CH}_{3}\right), 20.4\left(\mathrm{CH}_{3}\right), 17.7$ $\left(\mathrm{CH}_{3}\right) ; \mathrm{CG} \mathrm{t}_{\mathrm{R}}=15.6$ min.; LRMS (EI) m/z (\%)220 (33), 201 (12), 193 (25), 178 (47), 168 (5), 152 (77), 137 (47), 121 (35), 109 (100), 96 (44), 81 (92), 69 (86), 55 (35); HRMS (EI) calcd for $\mathrm{C}_{19} \mathrm{H}_{35} \mathrm{NOS}-\mathrm{C}_{4} \mathrm{H}_{8} 269.1813$, found 269.1819.

General procedure for the synthesis of sulfinamides 6 . To a dry flask were added $\left(R_{\mathrm{S}}\right)$ $N$-tert-butylsulfinamide $(\mathbf{1}, 61 \mathrm{mg}, 0.5 \mathrm{mmol})$ followed by indium powder $(71 \mathrm{mg}, 0.63$ mmol). The reaction vessel was evacuated and put under an Ar atmosphere. Then a solution of the corresponding ketone $(0.55 \mathrm{mmol})$ in dry THF $(1 \mathrm{~mL})$ and Ti(OEt $)_{4}(281$ $\mu \mathrm{L}, 1.25 \mathrm{mmol}$ ) were added successively and the reaction mixture was stirred under an Ar atmosphere for $12 \mathrm{~h}$ at $65{ }^{\circ} \mathrm{C}$. At this time 2,4-pentadienyl bromide (154 mg, 1.05 mmol) was added to the mixture and it was heated to $65^{\circ} \mathrm{C}$ for $7 \mathrm{~h}$. The mixture was allowed to reach room temperature and was carefully added over a stirring mixture of 4:1 EtOAc/brine $(20 \mathrm{~mL})$. The resulted white suspension was filtered through a short pad of Celite, washed with EtOAc and the organics were concentrated under reduced pressure. The resulted suspension was diluted in 4:1 EtOAc/Hexane $(20 \mathrm{~mL})$, filtered again through Celite and the organics were concentrated under reduced pressure.

$\left(R_{\mathrm{S}}, 3 R\right)$ - $N$-tert-Butylsulfinyl-3-methyl-4-vinylhex-5-en-3-amine $\quad(6 a) . \quad$ From 2butanone, the expected product was obtained following the general procedure as a colorless crystal (75 mg, 62\%, 97:3 dr according ${ }^{1} \mathrm{H}$ NMR) after column chromatography $\left(9: 1\right.$ Hexane/EtOAc): $\mathrm{mp} 35.2-36.8^{\circ} \mathrm{C} ;[\alpha]_{\mathrm{D}}{ }^{20}-66.6\left(c 0.72, \mathrm{CHCl}_{3}\right)$; 
$\mathrm{R}_{\mathrm{f}} 0.37$ (7:3 Hexane/EtOAc); IR v 3292, 3075, 2976, 2940, 1632, 1457, 1380, 1176, 1059, 1001, 919, $732 \mathrm{~cm}^{-1} ;{ }^{1} \mathrm{H}$ NMR (300 MHz, $\left.\mathrm{CDCl}_{3}\right) \delta 5.98-5.75$ (m, 2H), $5.24-$ $5.10(\mathrm{~m}, 4 \mathrm{H}), 3.49(\mathrm{~s}, 1 \mathrm{H}), 2.98(\mathrm{t}, J=8.6 \mathrm{~Hz}, 1 \mathrm{H}), 1.66(\mathrm{dq}, J=14.7,7.4 \mathrm{~Hz}, 1 \mathrm{H}), 1.56$ $(\mathrm{dq}, J=14.5,7.3 \mathrm{~Hz}, 1 \mathrm{H}), 1.29(\mathrm{~s}, 3 \mathrm{H}), 1.21(\mathrm{~s}, 9 \mathrm{H}), 0.87(\mathrm{t}, J=7.4 \mathrm{~Hz}, 3 \mathrm{H}) ;{ }^{13} \mathrm{C} \mathrm{NMR}$ $\left(101 \mathrm{MHz}, \mathrm{CDCl}_{3}\right) \delta 136.8(\mathrm{CH}), 136.2(\mathrm{CH}), 118.8\left(\mathrm{CH}_{2}\right), 118.1\left(\mathrm{CH}_{2}\right), 59.2(\mathrm{C}), 57.5$ $(\mathrm{CH}), 56.2(\mathrm{C}), 30.6\left(\mathrm{CH}_{2}\right), 24.1\left(\mathrm{CH}_{3}\right), 23.0\left(\mathrm{CH}_{3}\right), 7.5\left(\mathrm{CH}_{3}\right) ; \mathrm{GC} \mathrm{t}_{\mathrm{R}}=12.2$ min.; LRMS (EI) m/z (\%) 176 (16), 122 (5), 121 (6), 120 (100), 102 (21), 81 (10), 71 (5), 67 (10), 57 (17); HRMS (ESI) calcd for $\mathrm{C}_{13} \mathrm{H}_{26} \mathrm{NOS}\left(\mathrm{M}^{+}+1\right)$ 244.1735, found 244.1728.

$\left(R_{\mathrm{S}}, 4 R\right)$ - $N$-tert-Butylsulfinyl-4-methyl-3-vinylnon-1-en-4-amine $\quad(6 \mathrm{~b}) . \quad$ From 2heptanone $(75 \mu \mathrm{L}, 0.53 \mathrm{mmol})$, the expected product was obtained following the general procedure as a colorless oil (93 mg, 65\%, single diastereoisomer according to ${ }^{1} \mathrm{H} \mathrm{NMR}$ ) after column chromatography $\left(9: 1\right.$ Hexane/EtOAc): $[\alpha]_{\mathrm{D}}{ }^{20}-63.8\left(c 0.98, \mathrm{CHCl}_{3}\right) ; \mathrm{R}_{\mathrm{f}}$ 0.33 (7:3 Hexane/EtOAc); IR v 3297, 3075, 2954, 2935, 1632, 1456, 1380, 1178, 1064, 1002, 914, $731 \mathrm{~cm}^{-1} ;{ }^{1} \mathrm{H}$ NMR (300 MHz, $\left.\mathrm{CDCl}_{3}\right) \delta 5.93-5.78(\mathrm{~m}, 2 \mathrm{H}), 5.27-5.17$ $(\mathrm{m}, 2 \mathrm{H}), 5.16-5.10(\mathrm{~m}, 2 \mathrm{H}), 3.50(\mathrm{~s}, 1 \mathrm{H}), 2.97(\mathrm{t}, J=8.5 \mathrm{~Hz}, 1 \mathrm{H}), 1.67-1.43(\mathrm{~m}, 2 \mathrm{H})$, $1.40-1.30(\mathrm{~m}, 2 \mathrm{H}), 1.30(\mathrm{~s}, 3 \mathrm{H}), 1.28-1.20(\mathrm{~m}, 4 \mathrm{H}), 1.20(\mathrm{~s}, 9 \mathrm{H}), 0.88(\mathrm{t}, J=6.7 \mathrm{~Hz}$, $3 \mathrm{H}) ;{ }^{13} \mathrm{C}$ NMR $\left(101 \mathrm{MHz}, \mathrm{CDCl}_{3}\right) \delta 136.8(\mathrm{CH}), 136.1(\mathrm{CH}), 118.7\left(\mathrm{CH}_{2}\right), 118.1$ $\left(\mathrm{CH}_{2}\right), 59.0(\mathrm{C}), 57.7(\mathrm{CH}), 56.1(\mathrm{C}), 38.0\left(\mathrm{CH}_{2}\right), 32.2\left(\mathrm{CH}_{2}\right), 24.5\left(\mathrm{CH}_{3}\right), 22.9\left(\mathrm{CH}_{3}\right)$, $22.6\left(\mathrm{CH}_{2}\right), 22.5\left(\mathrm{CH}_{2}\right), 14.1\left(\mathrm{CH}_{3}\right) ; \mathrm{GC} \mathrm{t}_{\mathrm{R}}=13.9$ min.; LRMS (EI) $\mathrm{m} / \mathrm{z}(\%) 229$ (4), 163 (6), 162 (13), 161 (100), 159 (5), 158 (49), 144 (5), 118 (9), 110 (6), 105 (23), 97 (15), 95 (10), 91 (7), 67 (12), 57 (12), 55 (12); HRMS (ESI) calcd for $\mathrm{C}_{16} \mathrm{H}_{32} \mathrm{NOS}$ $\left(\mathrm{M}^{+}+1\right)$ 286.2205, found 286.2201.

$\left(R_{\mathrm{S}}, 3 R\right)$-N-tert-Butylsulfinyl-3-methyl-1-phenyl-4-vinylhex-5-en-3-amine (6c). From 4-phenyl-2-butanone, the expected product was obtained following the general procedure as a colorless oil $\left(115 \mathrm{mg}, 72 \%\right.$, single diastereoisomer according to ${ }^{1} \mathrm{H}$ 
NMR) after column chromatography (9:1 Hexane/EtOAc): $[\alpha]_{\mathrm{D}}{ }^{20}-74.2(c \quad 0.73$, $\left.\mathrm{CHCl}_{3}\right) ; \mathrm{R}_{\mathrm{f}} 0.34$ (7:3 Hexane/EtOAc); IR v 3076, 3025, 2977, 2953, 2867, 1632, 1603, 1455, 1381, 1063, 1063, 1002, $747 \mathrm{~cm}^{-1} ;{ }^{1} \mathrm{H}$ NMR (300 MHz, $\left.\mathrm{CDCl}_{3}\right) \delta 7.32-7.12(\mathrm{~m}$, 5H), $5.99-5.79(\mathrm{~m}, 2 \mathrm{H}), 5.34-5.11(\mathrm{~m}, 4 \mathrm{H}), 3.62(\mathrm{br} \mathrm{s}, 1 \mathrm{H}), 3.07(\mathrm{t}, J=8.6 \mathrm{~Hz}, 1 \mathrm{H})$, $2.73-2.54(\mathrm{~m}, 2 \mathrm{H}), 1.93(\mathrm{ddd}, J=14.2,10.9,6.4 \mathrm{~Hz}, 1 \mathrm{H}), 1.80(\mathrm{ddd}, J=14.2,11.2$, $7.0 \mathrm{~Hz}, 1 \mathrm{H}), 1.39$ (s, 3H), $1.24(\mathrm{~s}, 9 \mathrm{H}) ;{ }^{13} \mathrm{C} \mathrm{NMR}\left(101 \mathrm{MHz}, \mathrm{CDCl}_{3}\right) \delta 142.4(\mathrm{C}), 136.6$ $(\mathrm{CH}), 135.9(\mathrm{CH}), 128.6(\mathrm{CH}), 128.5(\mathrm{CH}), 126.1(\mathrm{CH}), 119.3\left(\mathrm{CH}_{2}\right), 118.4\left(\mathrm{CH}_{2}\right), 58.9$ (C), $58.0(\mathrm{CH}), 56.4(\mathrm{C}), 40.5\left(\mathrm{CH}_{2}\right), 29.5\left(\mathrm{CH}_{2}\right), 24.5\left(\mathrm{CH}_{3}\right), 23.1\left(\mathrm{CH}_{3}\right) ; \mathrm{GC} \mathrm{t}_{\mathrm{R}}=16.6$ min.; LRMS (EI) m/z (\%) 263 (8), 196 (7), 195 (56), 178 (5), 159 (14), 158 (38), 147 (47), 146 (21), 132 (15), 131 (25), 110 (29), 95 (6), 92 (9), 9 (100), 83 (18), 87 (11), 65 (11); HRMS (ESI) calcd for $\mathrm{C}_{19} \mathrm{H}_{30} \mathrm{NOS}\left(\mathrm{M}^{+}+1\right)$ 320.2048, found 320.2039.

$\left(R_{\mathrm{S}}, \mathbf{4 R}\right)$ - $N$-tert-Butylsulfinyl-3-ethyltridecan-4-amine (7). To a solution of compound 4f $(65 \mathrm{mg}, 0.20 \mathrm{mmol})$ in EtOAc $(6 \mathrm{~mL})$ was added $\mathrm{PtO}_{2}(6 \mathrm{mg}, 10 \mathrm{~mol} \%)$ and put under a hydrogen atmosphere. The mixture was vigorously stirred at room temperature for $15 \mathrm{~h}$. The catalyst was removed by filtration through a pad of Celite, eluting with more EtOAc. The solvent was removed under reduced pressure and the residue was purified by column chromatography (9:1 Hexane:EtOAc), to obtain the expected product as a colorless oil $(62 \mathrm{mg}, 93 \%):[\alpha]_{\mathrm{D}}{ }^{20}-38.6\left(c 0.79, \mathrm{CHCl}_{3}\right) ; \mathrm{R}_{\mathrm{f}} 0.54(7: 3$ Hexane/EtOAc); IR v 3243, 2957, 2923, 2871, 2854, 1462, 1362, 1056, $753 \mathrm{~cm}^{-1} ;{ }^{1} \mathrm{H}$ NMR (300 MHz, $\left.\mathrm{CDCl}_{3}\right) \delta 3.20(\mathrm{dt}, J=7.4,4.4 \mathrm{~Hz}, 1 \mathrm{H}), 3.02(\mathrm{~d}, J=7.6 \mathrm{~Hz}, 1 \mathrm{H}), 1.43$ $-1.30(\mathrm{~m}, 5 \mathrm{H}), 1.30-1.17(\mathrm{~m}, 16 \mathrm{H}), 1.14(\mathrm{~s}, 9 \mathrm{H}), 0.87(\mathrm{t}, J=7.3 \mathrm{~Hz}, 6 \mathrm{H}), 0.81(\mathrm{t}, J=$ $6.8 \mathrm{~Hz}, 3 \mathrm{H}) ;{ }^{13} \mathrm{C}$ NMR $\left(101 \mathrm{MHz}, \mathrm{CDCl}_{3}\right) \delta 58.4(\mathrm{CH}), 56.0(\mathrm{C}), 46.2(\mathrm{CH}), 32.4$ $\left(\mathrm{CH}_{2}\right), 32.0\left(\mathrm{CH}_{2}\right), 29.7\left(\mathrm{CH}_{2}\right), 29.7\left(\mathrm{CH}_{2}\right), 29.6\left(\mathrm{CH}_{2}\right), 29.4\left(\mathrm{CH}_{2}\right), 26.6\left(\mathrm{CH}_{2}\right), 22.9$ $\left(\mathrm{CH}_{3}\right), 22.8\left(\mathrm{CH}_{2}\right), 22.5\left(\mathrm{CH}_{2}\right), 22.1\left(\mathrm{CH}_{2}\right), 14.2\left(\mathrm{CH}_{3}\right), 12.4\left(\mathrm{CH}_{3}\right), 12.3\left(\mathrm{CH}_{3}\right) ; \mathrm{GC} \mathrm{t}_{\mathrm{R}}=$ 13.4 min.; LRMS (EI) m/z (\%) 203 (16), 186 (17), 157 (12), 156 (100), 154 (6), 100 
(12), 97 (7), 91 (33), 84 (11), 83 (19), 71 (11), 70 (14), 69 (12), 56 (15), 55 (19); HRMS (ESI) calcd for $\mathrm{C}_{19} \mathrm{H}_{42} \mathrm{NOS}\left(\mathrm{M}^{+}+1\right)$ 332.2987, found 332.2993.

$\left(R_{\mathrm{S}}, 3 R\right)$ - $N$-tert-Butylsulfinyl-4-ethyl-3-methylhexan-3-amine (8). Compound 8 was obtained from compound $\mathbf{6 a}(49 \mathrm{mg}, 0.2 \mathrm{mmol})$, following the same procedure used to obtain 7, as a colorless oil $(45 \mathrm{mg}, 92 \%):[\alpha]_{\mathrm{D}}{ }^{20}-55.7\left(c 0.79, \mathrm{CHCl}_{3}\right) ; \mathrm{R}_{\mathrm{f}} 0.34(7: 3$ Hexane/EtOAc); IR v 3237, 2961, 2875, 1464, 1379, 1362, 1178, 1052, 937, $920 \mathrm{~cm}^{-1}$; ${ }^{1} \mathrm{H}$ NMR (300 MHz, $\left.\mathrm{CDCl}_{3}\right) \delta 3.16$ (br s, 1H), $1.67-1.51(\mathrm{~m}, 4 \mathrm{H}), 1.35-1.26(\mathrm{~m}, 1 \mathrm{H})$, $1.22(\mathrm{~s}, 3 \mathrm{H}), 1.20(\mathrm{~s}, 9 \mathrm{H}), 1.19-1.08(\mathrm{~m}, 2 \mathrm{H}), 0.98(\mathrm{t}, J=7.3 \mathrm{~Hz}, 3 \mathrm{H}), 0.97(\mathrm{t}, J=7.3$ $\mathrm{Hz}, 3 \mathrm{H}), 0.88(\mathrm{t}, J=7.4 \mathrm{~Hz}, 3 \mathrm{H}) ;{ }^{13} \mathrm{C} \mathrm{NMR}\left(101 \mathrm{MHz}, \mathrm{CDCl}_{3}\right) \delta 61.7(\mathrm{C}), 55.9(\mathrm{C})$, $50.4(\mathrm{CH}), 31.4\left(\mathrm{CH}_{2}\right), 23.9\left(\mathrm{CH}_{3}\right), 23.1\left(\mathrm{CH}_{2}\right), 23.0\left(\mathrm{CH}_{2}\right), 22.9\left(\mathrm{CH}_{3}\right), 14.6\left(\mathrm{CH}_{3}\right)$, $14.0\left(\mathrm{CH}_{3}\right), 8.2\left(\mathrm{CH}_{3}\right) ; \mathrm{GC} t_{\mathrm{R}}=12.6$ min.; LRMS (EI) $\mathrm{m} / z(\%) 191$ (36), $176(19), 162$ (8), 127 (98), 126 (28), 120 (64), 119 (9), 102 (16), 97 (10), 85 (68), 72 (13), 71 (100), 57 (74), 55 (10); HRMS (ESI) calcd for $\mathrm{C}_{13} \mathrm{H}_{30} \mathrm{NOS}\left(\mathrm{M}^{+}+1\right)$ 248.2048, found 248.2037. $\left(R_{\mathrm{S}}, 4 R\right)$ - $N$-tert-Butylsulfinyl-1-hydroxyl-3-(2'-hydroxyethyl)-4-methylnonan-4amine (9b). The homoallylamine $6 \mathbf{b}(171 \mathrm{mg}, 0.6 \mathrm{mmol})$ was dissolved in dry THF $(0.2$ $\mathrm{mL})$ under an $\mathrm{Ar}$ atmosphere and cooled to $0^{\circ} \mathrm{C}$. A solution of 9-BBN (0.5 $\mathrm{M}$ in THF, $7.2 \mathrm{~mL}, 3.6 \mathrm{mmol}$ ), was added dropwise over ca. $10 \mathrm{~min}$. The stirring mixture was heated for $15 \mathrm{~h}$ at $60{ }^{\circ} \mathrm{C}$. After cooling to $0{ }^{\circ} \mathrm{C}$, a solution of $\mathrm{NaOH}(1.6 \mathrm{~mL}, 2 \mathrm{M})$ was carefully added and, after $5 \mathrm{~min}, \mathrm{H}_{2} \mathrm{O}_{2}$ solution $(30 \% \mathrm{wt} / \mathrm{v}, 1 \mathrm{~mL})$ was added. The mixture was stirred for $15 \mathrm{~h}$ at $60{ }^{\circ} \mathrm{C}$ and then cooled to room temperature. The organic phase was collected and the aqueous phase was extracted with EtOAc (x3). The organics were dried over $\mathrm{MgSO}_{4}$, filtered and concentrated to obtain the crude diol. After column chromatography $(98: 2 \mathrm{EtOAc} / \mathrm{MeOH})$ the pure product $\mathbf{9 b}$ was obtained as a colorless oil $\left(115 \mathrm{mg}, 60 \%\right.$, single diastereoisomer according to $\left.{ }^{1} \mathrm{H} \mathrm{NMR}\right):[\alpha]_{\mathrm{D}}{ }^{20}-$ 30.5 ( c 0.95, $\left.\mathrm{CHCl}_{3}\right) ; \mathrm{R}_{\mathrm{f}} 0.16(98: 2 \mathrm{EtOAc/MeOH}) ; \mathrm{IR} v 3301,2953,2933,2870,1457$, 
1363, 1098, 1012, 935, $753 \mathrm{~cm}^{-1} ;{ }^{1} \mathrm{H}$ NMR (300 MHz, $\left.\mathrm{CDCl}_{3}\right) \delta 3.82-3.71(\mathrm{~m}, 3 \mathrm{H})$, $3.71-3.53(\mathrm{~m}, 2 \mathrm{H}), 3.42($ br s, 1H), 3.12 (br s, 1H), $2.00-1.82(\mathrm{~m}, 4 \mathrm{H}), 1.54-1.31$ $(\mathrm{m}, 6 \mathrm{H}), 1.28(\mathrm{~s}, 3 \mathrm{H}), 1.27-1.23(\mathrm{~m}, 3 \mathrm{H}), 1.21(\mathrm{~s}, 9 \mathrm{H}), 0.89(\mathrm{t}, J=6.9 \mathrm{~Hz}, 3 \mathrm{H}) ;{ }^{13} \mathrm{C}$ NMR (101 MHz, $\left.\mathrm{CDCl}_{3}\right) \delta 62.3\left(\mathrm{CH}_{2}\right), 61.3(\mathrm{C}), 61.0\left(\mathrm{CH}_{2}\right), 56.1(\mathrm{C}), 41.5(\mathrm{CH}), 38.5$ $\left(\mathrm{CH}_{2}\right), 34.0\left(\mathrm{CH}_{2}\right), 33.4\left(\mathrm{CH}_{2}\right), 32.4\left(\mathrm{CH}_{2}\right), 23.3\left(\mathrm{CH}_{3}\right), 22.9\left(\mathrm{CH}_{3}\right), 22.8\left(\mathrm{CH}_{2}\right), 22.6$ $\left(\mathrm{CH}_{2}\right), 14.0\left(\mathrm{CH}_{3}\right) ; \mathrm{GC} \mathrm{t}_{\mathrm{R}}=15.8$ min.; LRMS (EI) $\mathrm{m} / \mathrm{z}(\%) 163$ (5), 161 (83), 160 (11), 134 (10), 129 (44), 128 (69), 115 (11), 114 (100), 112 (10), 111 (21), 110 (14), 105 (30), 91 (20), 85 (11), 84 (14), 83 (21), 82 (15), 81 (21), 71 (18), 70 (20), 69 (28), 67 (24), 57 (32), 55 (60); HRMS (ESI) calcd for $\mathrm{C}_{16} \mathrm{H}_{36} \mathrm{NO}_{3} \mathrm{~S}\left(\mathrm{M}^{+}+1\right)$ 322.2416, found 322.2418 .

$\left(R_{\mathrm{S}}, 3 R\right)$ - $N$-tert-Butylsulfinyl-1-hydroxyl-3-(2'-hydroxyethyl)-4-methyl-6-phenylhex4-amine (9c). From 6c $(191 \mathrm{mg}, 0.6 \mathrm{mmol})$, the expected product was obtained following the same procedure used for $\mathbf{9 b}$, as a colorless wax (136 $\mathrm{mg}$, 64\%, single diastereoisomer according to $\left.{ }^{1} \mathrm{H} \mathrm{NMR}\right)$ : $[\alpha]_{\mathrm{D}}{ }^{20}-39.9\left(c 0.80, \mathrm{CHCl}_{3}\right) ; \mathrm{R}_{\mathrm{f}} 0.15(98: 2$ EtOAc/MeOH); IR v 3271, 2949, 1454, 1363, 1031, $730 \mathrm{~cm}^{-1} ;{ }^{1} \mathrm{H}$ NMR $(300 \mathrm{MHz}$, $\left.\mathrm{CDCl}_{3}\right) \delta 7.33-7.27(\mathrm{~m}, 1 \mathrm{H}), 7.27-7.22(\mathrm{~m}, 1 \mathrm{H}), 7.22-7.09(\mathrm{~m}, 3 \mathrm{H}), 4.09(\mathrm{~s}, 1 \mathrm{H})$, $4.02-3.81(\mathrm{~m}, 1 \mathrm{H}), 3.81-3.72(\mathrm{~m}, 2 \mathrm{H}), 3.69-3.49(\mathrm{~m}, 2 \mathrm{H}), 2.74-2.54(\mathrm{~m}, 2 \mathrm{H}), 2.03$ $-1.84(\mathrm{~m}, 3 \mathrm{H}), 1.81-1.72(\mathrm{~m}, 2 \mathrm{H}), 1.49-1.39(\mathrm{~m}, 2 \mathrm{H}), 1.38(\mathrm{~s}, 3 \mathrm{H}), 1.23(\mathrm{~s}, 9 \mathrm{H}),{ }^{13} \mathrm{C}$ NMR (101 MHz, $\left.\mathrm{CDCl}_{3}\right) \delta 142.3(\mathrm{C}), 128.6(\mathrm{CH}), 128.4(\mathrm{CH}), 126.1(\mathrm{CH}), 62.1\left(\mathrm{CH}_{2}\right)$, $61.3(\mathrm{C}), 61.0\left(\mathrm{CH}_{2}\right), 56.3(\mathrm{C}), 41.4(\mathrm{CH}), 41.1\left(\mathrm{CH}_{2}\right), 34.0\left(\mathrm{CH}_{2}\right), 33.6\left(\mathrm{CH}_{2}\right), 29.9$ $\left(\mathrm{CH}_{2}\right), 23.4\left(\mathrm{CH}_{3}\right), 23.0\left(\mathrm{CH}_{3}\right) ; \mathrm{GC} \mathrm{t}_{\mathrm{R}}=17.22 \mathrm{~min}$; LRMS (EI) $\mathrm{m} / z(\%) 323(12), 289$ (10), 275 (20), 249 (13), 207 (8), 202 (14), 201 (82), 176 (11), 159 (41), 157 (11), 153 (17), 148 (12), 146 (15), 131 (34), 129 (100), 105 (32), 103 (11), 101 (54), 91 (72), 77 (14); HRMS (ESI) calcd for $\mathrm{C}_{19} \mathrm{H}_{34} \mathrm{NO}_{3} \mathrm{~S}\left(\mathrm{M}^{+}+1\right)$ 356.2259, found 356.2270. 
$\left(R_{\mathrm{S}}, 2 R, 3 R\right)$ - $N$-tert-Butylsulfinyl-2-methyl-2-pentyl-3-(2'-hydroxyethyl)pyrrolidine

(10b). The corresponding diol 9b (160 mg, $0.5 \mathrm{mmol})$ was dissolved in dry THF (1.7 $\mathrm{mL})$ under an $\mathrm{Ar}$ atmosphere and cooled to $0{ }^{\circ} \mathrm{C} . \mathrm{PPh}_{3}(157 \mathrm{mg}, 0.6 \mathrm{mmol})$ was added to the reaction mixture followed by a DIAD solution in THF $(1 \mathrm{~mL}, 0.6 \mathrm{M})$. The reaction was stirred for $15 \mathrm{~h}$ at $25^{\circ} \mathrm{C}$. All volatiles were removed under reduced pressure before purification by column chromatography $(99: 1$, EtOAc/MeOH) to obtain the corresponding pure products $10 \mathrm{~b}$ as a colorless oil (101 mg, 67\%, 96:4 dr crude, single stereoisomer after purification according $\left.{ }^{1} \mathrm{H} \mathrm{NMR}\right):[\alpha]_{\mathrm{D}}{ }^{20}-62.5\left(c\right.$ 1.05, $\left.\mathrm{CHCl}_{3}\right) ; \mathrm{R}_{\mathrm{f}}$ 0.29 (98:2 EtOAc/MeOH); IR v 3385, 2954, 2932, 2871, 1458, 1377, 1361, 1035, 1017 , $955 \mathrm{~cm}^{-1} ;{ }^{1} \mathrm{H}$ NMR $\left(300 \mathrm{MHz}, \mathrm{CDCl}_{3}\right) \delta 3.78(\mathrm{~m}, 2 \mathrm{H}), 3.65(\mathrm{dd}, J=16.0,8.2 \mathrm{~Hz}, 1 \mathrm{H})$, $2.78(\mathrm{dd}, J=16.6,9.7 \mathrm{~Hz}, 1 \mathrm{H}), 2.10-1.95(\mathrm{~m}, 2 \mathrm{H}), 1.91-1.45(\mathrm{~m}, 6 \mathrm{H}), 1.44-1.25$ $(\mathrm{m}, 6 \mathrm{H}), 1.22(\mathrm{~s}, 9 \mathrm{H}), 1.17(\mathrm{~s}, 3 \mathrm{H}), 0.89(\mathrm{t}, J=6.7 \mathrm{~Hz}, 3 \mathrm{H}) ;{ }^{13} \mathrm{C}$ NMR $(101 \mathrm{MHz}$, $\left.\mathrm{CDCl}_{3}\right) \delta 69.3(\mathrm{C}), 61.9\left(\mathrm{CH}_{2}\right), 57.4(\mathrm{C}), 41.2(\mathrm{CH}), 39.9\left(\mathrm{CH}_{2}\right), 39.8\left(\mathrm{CH}_{2}\right), 32.8$ $\left(\mathrm{CH}_{2}\right), 32.6\left(\mathrm{CH}_{2}\right), 29.5\left(\mathrm{CH}_{2}\right), 24.7\left(\mathrm{CH}_{3}\right), 23.3\left(\mathrm{CH}_{2}\right), 22.8\left(\mathrm{CH}_{2}\right), 21.7\left(\mathrm{CH}_{3}\right), 14.2$ $\left(\mathrm{CH}_{3}\right) ; \mathrm{GC} \mathrm{t}_{\mathrm{R}}=14.83$ min.; LRMS (EI) $\mathrm{m} / z(\%) 184$ (11), $166(10), 129$ (9), $128(100)$, 126 (11), 111 (16), 110 (14), 97 (10), 96 (14), 84 (11), 82 (13), 71 (12), 55 (15); HRMS (ESI) calcd for $\mathrm{C}_{16} \mathrm{H}_{34} \mathrm{NO}_{2} \mathrm{~S}\left(\mathrm{M}^{+}+1\right)$ 304.2310, found 304.2302.

$\left(R_{\mathrm{S}}, 2 R, 3 R\right)-N$-tert-Butylsulfinyl-3-(2-hydroxyethyl)-2-methyl-2-(2-

phenylethyl)pyrrolidine (10c). From compound 9c (106 mg, $0.3 \mathrm{mmol})$, the expected product was obtained following the same procedure to obtain compound $\mathbf{1 0 b}$, as a colorless oil (75 mg, 75\%, 96:4 dr crude, single diastereoisomer after purification according to ${ }^{1} \mathrm{H}$ NMR): $[\alpha]_{\mathrm{D}}{ }^{20}-40.4\left(\right.$ c $\left.1.10, \mathrm{CHCl}_{3}\right) ; \mathrm{R}_{\mathrm{f}} 0.21(98: 2 \mathrm{EtOAc/MeOH}) ; \mathrm{IR}$ $v 3370,3025,2960,1602,1455,1362,1031,750 \mathrm{~cm}^{-1} ;{ }^{1} \mathrm{H}$ NMR $\left(300 \mathrm{MHz}, \mathrm{CDCl}_{3}\right) \delta$ $7.37-7.24(\mathrm{~m}, 2 \mathrm{H}), 7.24-7.12(\mathrm{~m}, 3 \mathrm{H}), 3.82(\mathrm{t}, J=9.5 \mathrm{~Hz}, 1 \mathrm{H}), 3.79-3.68(\mathrm{~m}, 1 \mathrm{H})$, $3.62(\mathrm{dd}, J=15.8,8.5 \mathrm{~Hz}, 1 \mathrm{H}), 2.83(\mathrm{dd}, J=17.1,9.7 \mathrm{~Hz}, 1 \mathrm{H}), 2.67(\mathrm{t}, J=8.5 \mathrm{~Hz}, 2 \mathrm{H})$, 
$2.14(\mathrm{~s}, 1 \mathrm{H}), 2.08-1.94(\mathrm{~m}, 2 \mathrm{H}), 1.91-1.78(\mathrm{~m}, 2 \mathrm{H}), 1.78-1.58(\mathrm{~m}, 1 \mathrm{H}), 1.58-1.48$ $(\mathrm{m}, 1 \mathrm{H}), 1.47-1.35(\mathrm{~m}, 1 \mathrm{H}), 1.26(\mathrm{~s}, 9 \mathrm{H}), 1.22(\mathrm{~s}, 3 \mathrm{H}) ;{ }^{13} \mathrm{C} \mathrm{NMR}\left(101 \mathrm{MHz}, \mathrm{CDCl}_{3}\right) \delta$ $142.4(\mathrm{C}), 128.6(\mathrm{CH}), 128.4(\mathrm{CH}), 126.0(\mathrm{CH}), 69.3(\mathrm{C}), 61.6\left(\mathrm{CH}_{2}\right), 57.8(\mathrm{C}), 41.2$ $(\mathrm{CH}), 41.2\left(\mathrm{CH}_{2}\right), 40.0\left(\mathrm{CH}_{2}\right), 32.7\left(\mathrm{CH}_{2}\right), 29.9\left(\mathrm{CH}_{2}\right), 29.4\left(\mathrm{CH}_{2}\right), 24.9\left(\mathrm{CH}_{3}\right), 21.8$ $\left(\mathrm{CH}_{3}\right) ; \mathrm{GC} \mathrm{t}_{\mathrm{R}}=17.3$ min.; LRMS (EI) m/z (\%) 230 (9), 207 (17), 202 (25), 200 (21), 186 (24), 172 (12), 159 (36), 158 (32), 131 (24), 127 (43), 126 (41), 118 (16), 117 (25), 108 (11), 105 (10), 92 (11), 91 (100), 33 (65), 32 (71), 77 (11), 68 (17), 56 (17), 55 (24); HRMS (ESI) calcd for $\mathrm{C}_{19} \mathrm{H}_{32} \mathrm{NO}_{2} \mathrm{~S}\left(\mathrm{M}^{+}+1\right)$ 338.2154, found 338.2142.

(2R,3R)-N-benzoyl-3-(2-hydroxyethyl)-2-methyl-2-(2-phenylethyl)pyrrolidine (11). Pyrrolidine 10c $(20 \mathrm{mg}, 0.05 \mathrm{mmol})$ was dissolved in dry $\mathrm{MeOH}(0.5 \mathrm{~mL})$ at $0{ }^{\circ} \mathrm{C}$ and a $4 \mathrm{M}$ solution of $\mathrm{HCl}$ in dioxane $(50 \mu \mathrm{L})$ was added dropwise over $1 \mathrm{~min}$. After stirring for $1 \mathrm{~h}$, the solvent was removed under reduced pressure and the hydrochloride was dissolved in $\mathrm{CH}_{2} \mathrm{Cl}_{2}(1 \mathrm{~mL})$ and cooled to $0{ }^{\circ} \mathrm{C}$. A solution of $\mathrm{NaOH}(2 \mathrm{M}, 1 \mathrm{~mL})$ was added followed by benzoylchloride $(7 \mu \mathrm{L}, 0.06 \mathrm{mmol})$ and the reaction mixture was stirred at $25{ }^{\circ} \mathrm{C}$ for $15 \mathrm{~h}$. The product was extracted with $\mathrm{CH}_{2} \mathrm{Cl}_{2}$ and washed sequentially with $\mathrm{NaOH}(2 \mathrm{M})$ and brine. The organics were dried over $\mathrm{MgSO}_{4}$, filtered and concentrated under reduced pressure. After column chromatography (7:3 Hexane/EtOAc), the expected product 11 was obtained as a colorless oil (15 mg, 90\%, single diastereoisomer according to ${ }^{1} \mathrm{H}$ NMR after purification): $[\alpha]_{\mathrm{D}}{ }^{20}-61.5$ (c 1.00, $\left.\mathrm{CHCl}_{3}\right) ; \mathrm{R}_{\mathrm{f}} 0.31$ (1:1 Hexane/EtOAc); IR v 3406, 3025, 2930, 1612, 1415, $1265 \mathrm{~cm}^{-1}$; ${ }^{1} \mathrm{H}$ NMR $\left(300 \mathrm{MHz}, \mathrm{CDCl}_{3}\right) \delta 7.45-7.35(\mathrm{~m}, 5 \mathrm{H}), 7.31-7.27(\mathrm{~m}, 1 \mathrm{H}), 7.26-7.21(\mathrm{~m}$, 3H), $7.20-7.12(\mathrm{~m}, 1 \mathrm{H}), 3.79(\mathrm{dt}, J=15.6,6.1 \mathrm{~Hz}, 1 \mathrm{H}), 3.68(\mathrm{dt}, J=10.1,7.5 \mathrm{~Hz}, 1 \mathrm{H})$, $3.46-3.29(\mathrm{~m}, 2 \mathrm{H}), 2.97-2.81(\mathrm{~m}, 1 \mathrm{H}), 2.72-2.60(\mathrm{~m}, 2 \mathrm{H}), 2.37(\mathrm{dddd}, J=13.9$, 11.1, 5.8, 3.1 Hz, 1H), $2.01-1.90(\mathrm{~m}, 1 \mathrm{H}), 1.85-1.42(\mathrm{~m}, 5 \mathrm{H}), 1.38(\mathrm{~s}, 3 \mathrm{H}) ;{ }^{13} \mathrm{C} \mathrm{NMR}$ $\left(101 \mathrm{MHz}, \mathrm{CDCl}_{3}\right) \delta 169.8(\mathrm{C}), 142.6(\mathrm{C}), 139.0(\mathrm{C}), 129.4\left(\mathrm{CH}_{2}\right), 128.7\left(\mathrm{CH}_{2}\right), 128.5$ 
$\left(\mathrm{CH}_{2}\right), 128.5\left(\mathrm{CH}_{2}\right), 126.4\left(\mathrm{CH}_{2}\right), 125.9\left(\mathrm{CH}_{2}\right), 66.8(\mathrm{C}), 61.8\left(\mathrm{CH}_{2}\right), 50.9\left(\mathrm{CH}_{2}\right), 41.7$ $(\mathrm{CH}), 37.8\left(\mathrm{CH}_{2}\right), 32.0\left(\mathrm{CH}_{2}\right), 30.5\left(\mathrm{CH}_{2}\right), 28.6\left(\mathrm{CH}_{2}\right), 19.6\left(\mathrm{CH}_{3}\right) ; \mathrm{GC} \mathrm{t}_{\mathrm{R}}=22.5 \mathrm{~min}$; LRMS (EI) m/z (\%) 244 (01), 231 (23), 230 (39), 207 (28), 188 (14), 187 (12), 106 (9), 105 (100), 91 (9), 77 (25); HRMS (ESI) calcd for $\mathrm{C}_{22} \mathrm{H}_{28} \mathrm{NO}_{2}\left(\mathrm{M}^{+}+1\right)$ 338.2120, found 338.2129 .

(4R)-N-tert-Butylsulfonyl-1-hydroxyl-3-(2'-hydroxyethyl)-4-methylnonan-4-amine (12). The sulfinyl compound $9 \mathrm{~b}(112 \mathrm{mg}, 0.35 \mathrm{mmol})$ was dissolved in dry $\mathrm{CH}_{2} \mathrm{Cl}_{2}$ $(0.05 \mathrm{M})$ and placed under an Ar atmosphere. The solution was cooled at $0{ }^{\circ} \mathrm{C}$ and $\mathrm{m}$ CPBA (73 mg, $0.42 \mathrm{mmol}$ ) was added. The reaction was stirred $1 \mathrm{~h}$ at $0{ }^{\circ} \mathrm{C}$, observing full conversion by TLC. Quenched by adding a saturated aqueous solution of $\mathrm{NaHSO}_{3}$ and saturated aqueous solution of $\mathrm{NaHCO}_{3}$, the layers were separated and the aqueous phase was extracted with $\mathrm{CH}_{2} \mathrm{Cl}_{2}$. Combined organic extracts were dried over $\mathrm{MgSO}_{4}$, filtered and concentrated under reduced pressure. After column chromatography $(1: 1$ Hexane/EtOAc) the expected product was obtained as a colorless oil (112 mg, 95\%, single diastereoisomer according to $\left.{ }^{1} \mathrm{H} \mathrm{NMR}\right):[\alpha]_{\mathrm{D}}{ }^{20}-5\left(c 0.60, \mathrm{CHCl}_{3}\right) ; \mathrm{R}_{\mathrm{f}} 0.14(1: 1$ Hexane/EtOAc); IR v 3443, 2953, 2872, 1468, 1287, 1117, 1049, $735 \mathrm{~cm}^{-1} ;{ }^{1} \mathrm{H}$ NMR $\left(300 \mathrm{MHz}, \mathrm{CDCl}_{3}\right) \delta 4.37(\mathrm{~s}, 1 \mathrm{H}), 3.84-3.60(\mathrm{~m}, 4 \mathrm{H}), 2.01-1.74(\mathrm{~m}, 4 \mathrm{H}), 1.63-1.39$ $(\mathrm{m}, 4 \mathrm{H}), 1.38(\mathrm{~s}, 9 \mathrm{H}), 1.34(\mathrm{~s}, 3 \mathrm{H}), 1.31-1.16(\mathrm{~m}, 5 \mathrm{H}), 0.87(\mathrm{t}, J=6.8 \mathrm{~Hz}, 3 \mathrm{H}) ;{ }^{13} \mathrm{C}$ NMR (101 MHz, $\left.\mathrm{CDCl}_{3}\right) \delta 64.3(\mathrm{C}), 62.1\left(\mathrm{CH}_{2}\right), 61.3\left(\mathrm{CH}_{2}\right), 60.1(\mathrm{C}), 39.7(\mathrm{CH}), 38.9$ $\left(\mathrm{CH}_{2}\right), 33.2\left(\mathrm{CH}_{2}\right), 32.5\left(\mathrm{CH}_{2}\right), 32.3\left(\mathrm{CH}_{2}\right), 24.6\left(\mathrm{CH}_{3}\right), 23.1\left(\mathrm{CH}_{2}\right), 22.8\left(\mathrm{CH}_{2}\right), 21.5$ $\left(\mathrm{CH}_{3}\right), 14.3\left(\mathrm{CH}_{3}\right) ; \mathrm{GC} \mathrm{t}_{\mathrm{R}}=17.6$ min.; LRMS (EI) $\mathrm{m} / z(\%) 338\left(\mathrm{M}^{+}+1,1\right), 322(13)$, 241 (7), 234 (29), 202 (35), 115 (9), 114 (100), 57 (28); HRMS (ESI) calcd for $\mathrm{C}_{16} \mathrm{H}_{36} \mathrm{NO}_{4} \mathrm{~S}\left(\mathrm{M}^{+}+1\right)$ 338.2361, found 338.2357.

(2R,3R)- $N$-tert-Butylsulfonyl-2-methyl-2-pentyl-3-(2'-hydroxyethyl)pyrrolidine

(13). Compound 13 was obtained from compound $\mathbf{1 0 b}(90 \mathrm{mg}, 0.13 \mathrm{mmol})$ following 
the same procedure used to obtain compound 12. A single diastereoisomer was obtained as a colorless wax (40 mg, 95\%, 96:4 dr crude, single diastereoisomer according to ${ }^{1} \mathrm{H}$ NMR after purification): $[\alpha]_{\mathrm{D}}{ }^{20}-5\left(c 070, \mathrm{CHCl}_{3}\right) ; \mathrm{R}_{\mathrm{f}} 0.16(7: 3 \mathrm{Hexane} / \mathrm{EtOAc}) ; \mathrm{IR} v$ 3489, 2956, 2930, 2871, 1465, 1298, 1116, $752 \mathrm{~cm}^{-1} ;{ }^{1} \mathrm{H}$ NMR $\left(300 \mathrm{MHz}, \mathrm{CDCl}_{3}\right) \delta$ $3.83-3.73(\mathrm{~m}, 1 \mathrm{H}), 3.72-3.65(\mathrm{~m}, 1 \mathrm{H}), 3.64-3.54(\mathrm{~m}, 1 \mathrm{H}), 3.24(\mathrm{td}, J=9.9,6.5 \mathrm{~Hz}$, 1H), 2.99 (br s, 1H), $2.25-2.10(\mathrm{~m}, 1 \mathrm{H}), 2.10-1.89(\mathrm{~m}, 2 \mathrm{H}), 1.78-1.61(\mathrm{~m}, 2 \mathrm{H}), 1.61$ - $1.50(\mathrm{~m}, 1 \mathrm{H}), 1.50-1.42(\mathrm{~m}, 1 \mathrm{H}), 1.40(\mathrm{~s}, 9 \mathrm{H}), 1.37-1.34(\mathrm{~m}, 1 \mathrm{H}), 1.32(\mathrm{~s}, 3 \mathrm{H})$, $1.31-1.15(\mathrm{~m}, 5 \mathrm{H}), 0.88(\mathrm{t}, J=6.7 \mathrm{~Hz}, 3 \mathrm{H}) ;{ }^{13} \mathrm{C} \mathrm{NMR}\left(101 \mathrm{MHz}, \mathrm{CDCl}_{3}\right) \delta 72.1(\mathrm{C})$, $61.9(\mathrm{C}), 67.8\left(\mathrm{CH}_{2}\right), 49.8\left(\mathrm{CH}_{2}\right), 42.5(\mathrm{CH}), 39.5\left(\mathrm{CH}_{2}\right), 33.0\left(\mathrm{CH}_{2}\right), 32.4\left(\mathrm{CH}_{2}\right), 28.9$ $\left(\mathrm{CH}_{2}\right), 25.6\left(\mathrm{CH}_{3}\right), 24.33\left(\mathrm{CH}_{2}\right), 22.8\left(\mathrm{CH}_{2}\right), 22.6\left(\mathrm{CH}_{3}\right), 14.2\left(\mathrm{CH}_{3}\right) ; \mathrm{GC} \mathrm{t}_{\mathrm{R}}=18.0$ min.; LRMS (EI) m/z (\%) 248 (13), 184 (7), 129 (8), 128 (100), 111 (6), 57 (22); HRMS (ESI) calcd for $\mathrm{C}_{16} \mathrm{H}_{34} \mathrm{NO}_{3} \mathrm{~S}\left(\mathrm{M}^{+}+1\right) 320.2259$, found 320.2270 .

$(2 R, 3 S)-$

and

$(2 R, 3 R)-N$-tert-Butylsulfonyl-2-methyl-2-pentyl-3-(2'hydroxyethyl)pyrrolidine (13 and 14). A 1:1 mixture of compounds 13 and $\mathbf{1 4}$ was obtained from compound $12(100 \mathrm{mg}, 0.15 \mathrm{mmol})$ following the same procedure described for 10b, as a colorless oil (47 mg, 50\% yield). ${ }^{1} \mathrm{H} \mathrm{NMR}\left(300 \mathrm{MHz}, \mathrm{CDCl}_{3}\right) \delta$ $3.85-3.72(\mathrm{~m}, 2 \mathrm{H}), 3.71-3.53(\mathrm{~m}, 4 \mathrm{H}), 3.44(\mathrm{dd}, J=16.4,8.1 \mathrm{~Hz}, 1 \mathrm{H}, 14), 3.24(\mathrm{dd}, J$ $=16.3,9.8 \mathrm{~Hz}, 1 \mathrm{H}, 13), 2.25-2.10(\mathrm{~m}, 1 \mathrm{H}), 2.10-1.94(\mathrm{~m}, 4 \mathrm{H}), 1.93-1.46(\mathrm{~m}, 10 \mathrm{H})$, $1.45(\mathrm{~s}, 3 \mathrm{H}, \mathbf{1 4}), 1.40(\mathrm{~s}, 18 \mathrm{H}), 1.32(\mathrm{~s}, 3 \mathrm{H}, \mathbf{1 3}), 1.32-1.01(\mathrm{~m}, 11 \mathrm{H}), 0.88(\mathrm{t}, J=6.5$ $\mathrm{Hz}, 6 \mathrm{H})$.

(4R)-N-tert-Butylsulfonyl-1-(4'-oxacyclohexyl) heptan-2-amine (15). Compound 15 (20\%) was obtained as byproduct in the reaction to obtain compounds $\mathbf{1 3}$ and $\mathbf{1 4} . \mathrm{R}_{\mathrm{f}}$ 0.50 (7:3 Hexane/EtOAc); IR $v 3289,2952,2928,1457,1299,1121,952 \mathrm{~cm}^{-1} ;{ }^{1} \mathrm{H}$ NMR (300 MHz, $\left.\mathrm{CDCl}_{3}\right) \delta 4.11-3.98(\mathrm{~m}, 2 \mathrm{H}), 3.48-3.29(\mathrm{~m}, 3 \mathrm{H}), 1.88(\mathrm{tt}, J=12.1$, $3.2 \mathrm{~Hz}, 1 \mathrm{H}), 1.78-1.44(\mathrm{~m}, 7 \mathrm{H}), 1.40(\mathrm{~s}, 9 \mathrm{H}), 1.35(\mathrm{~s}, 3 \mathrm{H}), 1.33-1.16(\mathrm{~m}, 7 \mathrm{H}), 0.90$ 
$(\mathrm{t}, J=6.8 \mathrm{~Hz}, 3 \mathrm{H}) ;{ }^{13} \mathrm{C}$ NMR $\left(101 \mathrm{MHz}, \mathrm{CDCl}_{3}\right) \delta 68.4(\mathrm{C}), 63.5\left(\mathrm{CH}_{2}\right), 60.3\left(\mathrm{CH}_{2}\right)$, $53.6(\mathrm{C}), 44.9(\mathrm{CH}), 38.4\left(\mathrm{CH}_{2}\right), 32.3\left(\mathrm{CH}_{2}\right), 27.8\left(\mathrm{CH}_{2}\right), 27.3\left(\mathrm{CH}_{2}\right), 24.7\left(\mathrm{CH}_{3}\right), 23.2$ $\left(\mathrm{CH}_{2}\right), 22.8\left(\mathrm{CH}_{2}\right), 21.2\left(\mathrm{CH}_{3}\right), 14.2\left(\mathrm{CH}_{3}\right) ; \mathrm{GC} \mathrm{t}_{\mathrm{R}}=16.6 \mathrm{~min}$; $\mathrm{LRMS}(\mathrm{EI}) \mathrm{m} / z(\%) 248$ (15), 234 (30), 178 (7), 128 (43), 114 (100), 57 (39); HRMS (ESI) calcd for $\mathrm{C}_{16} \mathrm{H}_{34} \mathrm{NO}_{3} \mathrm{~S}\left(\mathrm{M}^{+}+1\right) 320.2259$, found 320.2263.

\section{ACKNOWLEDGMENT}

We thank the Spanish Ministerio de Ciencia e Innovación (CTQ2011-24165) for financial support. I. B. acknowledges the Generalitat Valenciana for a predoctoral fellowship (ACIF/2011/159). E. B. acknowledges the Council of Higher EducationTurkey for a postdoctoral fellowship (16.10.12-B.09.6.YÖK.0.71.01-207.02-12285).

Supporting Information: Copies of ${ }^{1} \mathrm{H}$ and ${ }^{13} \mathrm{C}$ NMR spectra for compounds 3-15, and crystallographic data for compound 4e (CDCC 971541). This material is available free of charge via the Internet at http://pubs.acs.org.

\section{REFERENCES}

(1) Yasuda, H.; Yamauchi, M.; Nakamura, A.; Sei, T.; Kai, Y.; Yasuoka, N.; Kasai, N. Bull. Chem. Soc. Jpn. 1980, 53, 1089.

(2) Yasuda, H.; Ohnuma, Y.; Nakamura, A.; Sei, T.; Kai, Y.; Yasuoka, N., Kasai, N. Bull. Chem. Soc. Jpn. 1980, 53, 1101.

(3) (a) Ghosez, L.; Marko, I.; Hesbain-Frisque, A.-M. Tetrahedron Lett. 1986, 27, 5211.

(b) Jung, M. E.; Nichols, C. Tetrahedron Lett. 1996, 37, 7667. (c) Grilli, S.; Martelli, 
G.; Savoia, D. Eur. J. Org. Chem. 2001, 2917. (d) Alvaro, G.; Grepioni, F.; Grilli, S.; Maini, L.; Martelli, G.; Savoia, D. Synthesis, 581.

(4) Nishigaichi, Y.; Fujimoto, M.; Takuwa, A. Synlett 1994, 731. (b) Yanagisawa, A.; Nakatsuka, Y.; Nakashima, H.; Yamamoto, H. Synlett 1997, 933.

(5) Hosomi, A.; Saito, M.; Sakurai, H. Tetrahedron Lett. 1980, 21, 3783.

(6) (a) Fujita, K.; Schlosser, M. Helv. Chim. Acta 1982, 65, 1258. (b) Suginome, M.; Yamamoto, Y.; Fujii, K.; Ito, Y. J. Am. Chem. Soc. 1995, 117, 9608.

(7) (a) Murakami, M.; Anderson, P. G.; Suginome, M.; Ito, Y. J. Am. Chem. Soc. 1991, 113, 3987. (b) Suginome, M.; Yamamoto, Y.; Fujii, K.; Ito, Y. J. Am. Chem. Soc. 1995, 117, 9608. See also reference 3.

(8) Nishigaichi, Y.; Ishihara, M.; Fushitani, S.; Uenaga, K.; Takuwa, A. Chemistry Lett. 2004, 33, 108.

(9) (a) For a recent review on the application of organoindium compounds, see: Shen, Z.-L.; Wang, S.-Y.; Chok, Y.-K.; Xu, Y.-H.; Loh, T.-P. Chem. Rev. 2013, 113, 271. (b) For a review on the indium-mediated allylation of sulfinyl aldimines, see: Lin, G.-Q.; Xu, M.-H.; Zhong, Y.-W.; Sun, X.-W. Acc. Chem. Res. 2008, 41, 831.

(10) Hirashita, T.; Inoue, S.; Yamamura, H.; Kawai, M.; Araki, S. J. Organomet. Chem. 1997, 549, 305.

(11) (a) Woo, S.; Squires, N.; Fallis, A. G. Org. Lett. 1999, 1, 573. (b) Melekhov, A.; Fallis, A. G. Tetrahedron Lett. 1999, 40, 7867.

(12) Cheng, B.; Sunderhaus, J. D.; Martin, S. F. Org. Lett. 2010, 12, 3622.

(13) During the preparation of this manuscript the diastereoselective addition of a pentadienyltin reagent to racemic aryl sulfinyl imines was reported. Notably, under the conditions used to obtain the $\gamma$-adduct with high diastereoselectivity ( 2 equivalents of $\mathrm{InCl}_{3}$ in $\mathrm{CH}_{2} \mathrm{Cl}_{2}$ at $-43{ }^{\circ} \mathrm{C}$ ), the only tert-butylsulfinyl imine examined was completely 
unreactive. See: Nishigaichi, Y.; Tsuruta, S.; Uenaga, K.; Awamura, T.; Iwamoto, H.; Takuwa, A. Tetrahedron Lett. 2014, 55, 510.

(14) (a) González-Gómez, J. C.; Medjahdi, M.; Foubelo, F.; Yus, M. J. Org. Chem. 2010, 75, 6308. (b) González-Gómez, J. C.; Foubelo, F.; Yus, M. Org. Synth. 2012, 89, 88.

(15) For comprehensive reviews on the application of tert-butylsulfinamides, see: (a) Ferreira, F.; Botuha, C.; Chemla, F.; Perez-Luna, A. Chem. Soc. Rev. 2009, 38, 1162. (b) Ellman, J. A.; Robak, M. T.; Herbage, M. A. Chem. Rev. 2010, 110, 3600.

(16) To inequivocally assign the signals of the minor diastereoisomer in the ${ }^{1} \mathrm{H}-\mathrm{NMR}$ spectra we have prepared authentic samples of diastereomeric mixtures for compounds $\mathbf{4 a}, \mathbf{4 e}, \mathbf{4 f}, \mathbf{4 h}, \mathbf{4 i}$, and $\mathbf{4 j}$ (see SI) according to a reported procedure: Brak, K.; Barrett, K. T.; Ellman, J. A. J. Org. Chem. 2009, 74, 3606.

(17) Sirvent, J. A.; Foubelo, F.; Yus, M. Chem. Commun. 2012, 48, 2543.

(18) Unfortunately, methylphenyl ketone and $\alpha, \beta$-unsaturated ketones (like 4-phenyl-3buten-2-one and 2-cyclohexenone) failed to give the expected adduct, affording either the corresponding ketimine or complex mixtures of products.

(19) The Lewis acid-catalyzed $E / Z$ isomerization of tert-butylsulfinyl ketimines has been already reported. See: Cogan, D. A.; Liu, G.-C.; Ellman, J. Tetrahedron 1999, 55, 8883.

(20) This dynamic kinetic resolution was also observed in the simple indium-mediated allylation of isolated tert-butylsulfinyl ketimines and we assigned the absolute configurations of compounds $\mathbf{6 a}, \mathbf{6 b}$ and $\mathbf{6 c}$ assuming the same mode of addition (see Reference 17).

(21) In these hypothetical transition states we did not define the geometry of the internal double-bond of the pentadienyl moiety because it is not relevant for the stereochemical 
outcome of the reaction. In the case of aldimines it seems more reasonable to place the vinylic group in an equatorial position; however gauche interactions along $\mathrm{C} 2-\mathrm{C} 3$ are more severe with ketimines. In fact, during the addition of pentadienyl indium reagents to $\alpha, \beta$-unsaturated ketones it was proposed a transition state where the vinylic group occupies an axial position; see: Villalva,-Servín, N. P.; Melekov, A.; Fallis, A. G. Synthesis 2003, 5, 790.

(22) Procupiou, G.;Lewis, W.;Harbottle, G.; Stockman, R. A. Org. Lett. 2013, 15, 2030.

(23) For precedents on pyrrolidine ring formation by Mitsunobu cyclization of tertbutylsulfinamines, see: (a) Jakobsche, C. E.; Peris, G.; Miller, S. J. Angew. Chem. Int. Ed. 2008, 47, 6707. (b) Hahn, K. N.; Fadeyi, O. O.; Cho, H. P.; Lindsley, C. W. Tetrahedron Lett. 2012, 53, 3577.

(24) After column purification of $\mathbf{1 0 b}$ and $\mathbf{1 0 c}$, a mixed fraction of the minor isomer and the major one facilitated the identification of the ${ }^{1} \mathrm{H}-\mathrm{NMR}$ signals of the minor diastereoisomers in order to calculate the diastereoselectivity.

(25) Paddon-Row, M. N.; Longshaw, A. I.; Willis, A. C.; Sherburn, M. S. Chem. Asian J. 2009, 4, 126.

(26) 5-Bromopentanal was prepared from ethyl 5-bromovaleronate by DIBAL-H reduction at $-78^{\circ} \mathrm{C}$. 